\title{
A multiplex microchamber diffusion assay for the antibody- based detection of microRNAs on randomly ordered microbeads
}

\author{
Christiane Geithe $^{1,6}$, Bo Zeng ${ }^{1,7}$, Carsten Schmidt ${ }^{1}$, Franziska Dinter ${ }^{1}$, Dirk \\ Roggenbuck $^{1,2,6}$, Werner Lehmann ${ }^{3}$, Gregory Dame $^{4}$, Peter Schierack ${ }^{1,6}$, Katja Hanack $^{5}$ \\ and Stefan Rödiger ${ }^{1,6}$
}

1 BTU Cottbus-Senftenberg, Institute of Biotechnology, Senftenberg, Germany

2 GA Generic Assays GmbH, Blankenfelde-Mahlow, Germany

3 Attomol Molekulare Diagnostika GmbH, Bronkow, Germany

4 Brandenburg Medical School Theodor Fontane, Institute of Microbiology and Virology, Campus Senftenberg, Senftenberg, Germany

5 University of Potsdam, Institute of Biochemistry and Biology, Potsdam-Golm, Germany

6 Faculty of Health Sciences Brandenburg, Senftenberg, Germany

7 Chinese Academy of Sciences, Institute of Biophysics, Beijing, China

Corresponding author:

Stefan Rödiger

Email address: stefan.roediger@b-tu.de

Katja Hanack

Email address: katja.hanack@uni-potsdam.de 


\begin{abstract}
Background: MicroRNAs (miRNAs) are small, conserved, noncoding RNAs regulating gene expression that functions in RNA silencing and post-transcriptional regulation of gene expression. Altered miRNA profiles have been implicated in many human diseases, and due to their circulating abilities, they have excited great interest in their use as clinical biomarkers. The development of innovative methods for miRNA detection has become of high scientific and clinical interest.

Methods: We developed a diffusion-driven microbead assay and combined it with an antibody-based miRNA detection. The diffusion process was carried out in two different approaches a) co-diffusion of miRNA and antibodies (termed diffusion approach I, DAI) and b) diffusion of miRNA in an antibody-saturated environment (DAII). In both approaches, neutravidin-coated microbeads were loaded with specific biotinylated DNA capture probes, which targets either miR-21-5p, miR-30a-3p or miR-93-5p. The miRNAs were time- and dose-dependently detected in a diffusion microchamber by primary anti-DNA:RNA hybrid and fluorescence-labeled secondary antibodies using our in-house developed inverse fluorescence microscope imaging platform VideoScan.

Results: Our assay offers the advantage that several target molecules can be detected simultaneously and in real-time in one reaction environment (multiplex), without any amplification steps. We recorded the diffusion process over a period of $24 \mathrm{~h}$ and found that the reaction was almost completed after $2 \mathrm{~h}$. The specificity of the assay was $96.7 \%$ for DAI and $92.3 \%$ for DAII. The detection limits were in a concentration range of $0.03-0.43 \mathrm{nM}$ for DAI and 0.14-1.09 nM for DAII, depending on the miRNA.

Conclusion: The miRNAs are successively exposed to the capture probe-loaded randomly ordered microbeads ( $\mathrm{p}$ value of CSR 0.23-0.96), which leads to microbeads that become saturated with the target molecules first in front rows. Non-bonded miRNAs continue to diffuse further and can therefore subsequently bind to the microbeads with free binding sites. Our detection principle differs from other microbead assays, in which all microbeads are simultaneously mixed with the sample solution, so that all target molecules bind equally distributed to the microbeads, resulting in an averaged signal intensity.
\end{abstract}

Keywords: microRNA, diffusion assay, microbeads, microchamber, antibodies 


\section{Introduction}

MicroRNAs (miRNAs) are short (20-22 nucleotides), highly conserved, noncoding RNAs, which regulate gene expression post-transcriptionally by targeting mRNAs, thus leading to degradation of mRNA or inhibition of translation. They have been identified as biomolecules that are involved in the development of many human diseases, such as cancer, inflammatory diseases, neurodegenerative diseases or immune-related diseases [1, 2].

The miRNAs were also associated with heart disease [3-5]. Heart disease is one of the most widespread causes of death in western countries. The dilated cardiomyopathy (DCM) is a major contributor to heart disease. DCM has a mortality rate of $50 \%$ within five years and is the most common cause of heart transplantation. Characteristics for DCM are the strong volume increase (dilatation) of the left and right ventricles and a homogeneous contractile dysfunction of the myocardium with the consequence of a restricted systolic function [6-9].

Due to the ability of miRNAs to circulate in body fluids such as blood, plasma or serum, and their stability therein, they emerged as potent biomarkers for diagnosis and prognosis [10, 11]. Altered miRNA profiles associated with certain diseases can be identified by technologies such as high-throughput sequencing, microarrays or quantitative PCR (qPCR) analysis [11-13]. It is also generally accepted that amplification-based systems can be biased, as amplification can lead to a shift in certain target molecules [14]. To reduce technical demands, costs and hands-on time, the development of alternative methods for miRNA detection has become a field of intensive research and clinical interest. Amplification-free detection systems in particular are repeatedly the subject of research work. In the assay described by Kappel et al. [15], for example, biotinylated complementary capture probes are hybridized directly with miRNAs and then the resulting hybrids are bound to a solid phase. Optical detection is performed with an anti-DNA:RNA hybrid antibody labeled with an acridinium ester, resulting in subsequent enzymatic signal amplification [15]. In contrast, the nanostring technology uses two complementary probes for hybridization, a target specific biotinylated capture probe and a target specific 
barcoded fluorescence reporter probe. The unique target-probe complex is immobilized and aligned on the imaging surface for the fluorescence readout [16].

Optical methods are common and widely used for the detection of biomolecules or especially miRNAs. We used our in-house developed VideoScan platform [17] as a readout platform, which is based on a fully automated multispectral fluorescence microscope. For this, we have developed multiplex assays for the imaging platform to detect and quantify biomolecules including short protein or DNA sequences [18] and (auto)antibodies [19]. The platform is also suitable for real-time measurements such as qPCRs, isothermal amplification and melting curve analysis [20, 21]. Microbead-based assays offer the advantage that several target molecules can be detected and quantified simultaneously in a reaction environment (multiplex). Planar microarrays have disadvantages regarding the mechanisms of surface interactions. In particular, the mass transport on the surface is limited [22]. Especially for the detection of short single-stranded molecules there are a number of detection principles based on molecular beacons and FRET probes [23]. Their detection principle is based on microbeads that have specific capture probes on their surface to which the target molecules can bind. All microbeads are simultaneously (bulk) mixed with the sample solution, so that in principle all target molecules can bind equally distributed to the microbeads. The binding event is then quantified by determining the surface signal intensity of all microbeads.

An alternative approach is that the target molecules are successively exposed to the capture probe-loaded microbeads. One can postulate as a hypothesis that in this approach the capture probe-loaded microbeads are saturated with the target molecules. Non-bonded target molecules can therefore subsequently bind to the microbeads with free binding sites. Based on this hypothesis, we have developed a microchamber in which the target molecules (miRNAs) diffuse through a field of capture probe-loaded microbeads and govern with their binding partners. The aim of this study was to design an antibody-based amplification-free assay that does not require labelling of miRNAs and can detect multiple molecules simultaneously (multiplex) at a defined temperature (isothermal). A further criterion was a stepwise and real-time resolved visualization of the binding process between the target nucleic acids and their capture molecules. 


\section{Methods}

\section{Assembly of the diffusion microchamber}

We used hybriwell chambers ( 16 wells, $7 \mathrm{~mm}$ x $7 \mathrm{~mm}$ x $0.05 \mathrm{~mm}$; RD477991, Grace BioLabs, Oregon, USA) that were customized according to our needs. To make the hybriwell chamber suitable for long-time experiments, we have modified them with reaction reservoirs at the inlets and outlets (Fig. 1). First, the adhesive hybriwell chamber (Fig. 1A) was glued to a glass slide. To modify the chamber, PCR reaction vessels were cut to a size of about $6 \mathrm{~mm}$ by cutting off the upper and lower ends using a custom-made cutting device (Fig. 1B, C). The resulting truncated hollow cones were then attached around the chamber inlets and outlets using a mixture of UV adhesive and carbon black. The carbon black was used to prevent the auto-fluorescence of the UV adhesive. The adhesive mixture was cured by UV irradiation ( $2 \times 3 \mathrm{~min}$ at $312 \mathrm{~nm}$ ) in a UV cross-linker (BIO-Link BLX-E, Vilber Lourmat, Germany). The modified microchamber consists of 16 cavities, each with a reservoir at the inlet and outlet (Fig. 1D). The attached reservoirs were used to seal the reaction chamber airtight to prevent evaporation or formation of air bubbles that could impair the diffusion process.

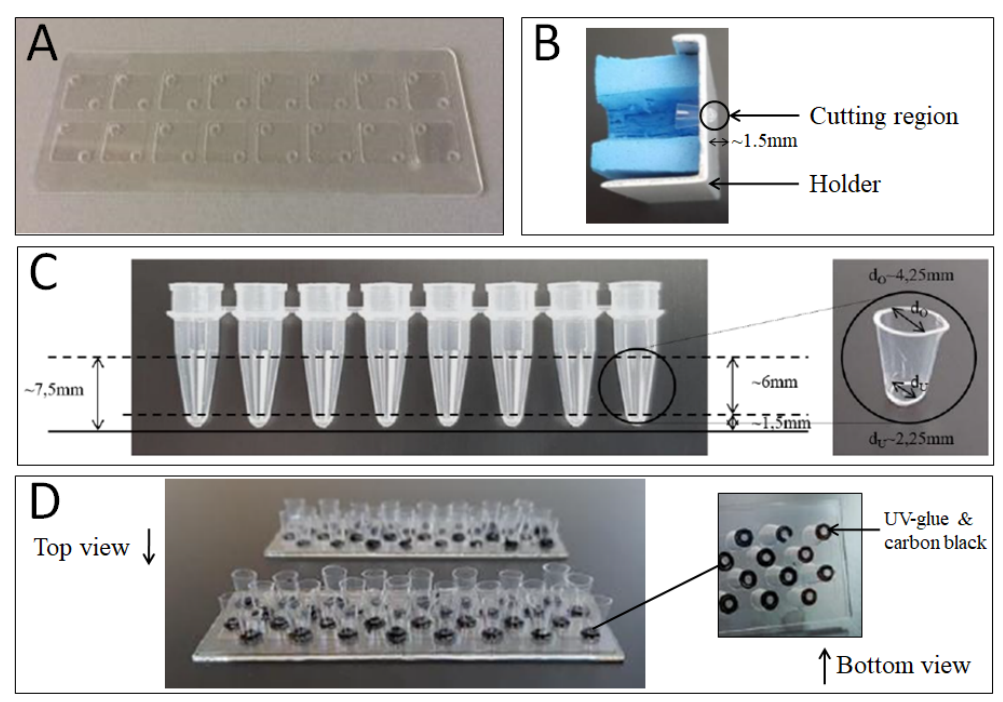

Figure 1. Modification of the hybriwell chamber. (A) GraceBio-Labs hybriwell chamber, (B) custom-made cutting device for precisely cutting the reaction vessels, (C) cutting of the reaction vessels to size, (D) modified microchamber from top and bottom. 


\section{Immobilizing of miRNA-specific capture probes on microbeads}

\section{Microbeads}

We used three different carboxylated poly(methyl methacrylate) (PMMA) microbead (MB) populations (PolyAn GmbH, Germany) which differ in size (MB1: $11 \mu \mathrm{m}, \mathrm{MB} 2: 11.5 \mu \mathrm{m}$, MB3: $12 \mu \mathrm{m}$ ) and a specific ratio of two fluorescence dyes (rhodamine 6G, excitation wavelength Ex 520 nm, emission wavelength Em 560 nm; coumarin 6, Ex 460 nm, Em 500 $\mathrm{nm})$.

\section{Covalent coupling of neutravidin}

The size/dye-encoded microbeads were coated with neutravidin (Thermo Fisher Scientific, Germany) by covalent coupling using 1-ethyl-3-(3-dimethylaminopropyl) carbodiimide hydrochloride (EDC; Karl Roth, Germany) cross-linker according to [24]. Neutravidin was used because it has a high temperature stability and a high binding constant [25]. In brief, $1.5 \times 10^{5}$ microbeads were first washed with $200 \mu \mathrm{L} 0.1 \mathrm{M}$ 2-(N-morpholino) ethanesulfonic acid (MES, pH 4.5; Karl Roth, Germany) and incubated for $30 \mathrm{~min}$ at $28^{\circ} \mathrm{C}$ and $1,200 \mathrm{rpm}$ with $100 \mu \mathrm{L}$ EDC solution ( $25 \mathrm{mg} / \mathrm{mL}$ EDC dissolved in MES). The microbeads were then washed with $200 \mu \mathrm{L} 0.05 \mathrm{x}$ phosphate buffered saline (PBS) and incubated for $3 \mathrm{~h}$ at $28^{\circ} \mathrm{C}$ and 1,200 rpm with $50 \mu \mathrm{L}$ neutravidin solution ( $60 \mu \mathrm{g} / \mathrm{mL}$ dissolved in $0.05 \times \mathrm{PBS})$. This was followed by three washing steps with Tween-20 containing Tris-buffered saline (TBST; $50 \mathrm{mM}$ Tris, $154 \mathrm{mM} \mathrm{NaCl}, 0.01 \%$ Tween-20, $\mathrm{pH}$ 7.4). Coupling of miRNA-specific capture probes

Dual-biotinylated miRNA-specific DNA capture probes (50 nM; complementary DNA sequence to the respective target) for hsa-miR-21-5p (5'-TCA ACA TCA GTC TGA TAA GCT A-dual-Biotin-3'), hsa-miR-30a-3p (5'-GCT GCA ACA TCC GAC TGA AAG-dual-Biotin-3') or hsa-miR-93-5p (5'-CTA CCT GCA CGA ACA GCA CTT TG-dual-Biotin-3'; Biomers.net, Germany) were each immobilized on a different neutravidin-coated microbead population. The reaction was conducted in a volume of $50 \mu \mathrm{L}$ for $15 \mathrm{~min}$ at room temperature under vigorous shaking $(1,200 \mathrm{rpm})$ in TBS-T followed by three times wash with PBS containing Tween-20 (PBS-T; $137 \mathrm{mM} \mathrm{NaCl}, 2.7 \mathrm{mM} \mathrm{KCl}, 10 \mathrm{mM} \mathrm{Na}_{2} \mathrm{HPO}_{4}, 2 \mathrm{mM} \mathrm{KH}_{2} \mathrm{PO}_{4}, 0.01 \%$ 
Tween-20, pH 7.4). Afterwards, all three capture probe-loaded microbead populations were bulk mixed (3-plex) in comparable proportions (approx. 1,000 beads per population).

\section{Sample preparation for the multiplex microbead-based miRNA detection by antibodies in a diffusion microchamber}

\section{Diffusion approach I - co-diffusion of miRNAs and antibodies}

The procedure of sample preparation is illustrated in Figure 2. First, a $7 \mu \mathrm{L}$ suspension containing three different microbead populations (3-plex, approx. 1,000 beads per population) with specific DNA capture probes (cp) for either hsa-miR-21-5p, hsa-miR-30a$3 p$ or hsa-miR-93-5p were added from one side of the microchamber and distributed randomly over the entire bottom surface (Fig. 2A). The miRNAs ( $0.05 \mathrm{nM}, 0.1 \mathrm{nM}, 0.5 \mathrm{nM}, 1$ nM, 5 nM, $10 \mathrm{nM}$ or $50 \mathrm{nM}$ hsa-miR-21-5p: 5'-UAG CUU AUC AGA CUG AUG UUG A-3', hsamiR-30a-3p: 5'-CUU UCA GUC GGA UGU UUG CAG C-3' or hsa-miR-93-5p: 5'-CAA AGU GCU GUU CGU GCA GGU AG-3'; Biomers.net, Germany) to be detected, the primary antibody (2.0 $\mu \mathrm{g} / \mathrm{mL}$; anti-DNA:RNA hybrid, clone S9.6; monoclonal, mouse, IgG2ak, Merck Millipore, Germany) and the secondary fluorescence-labeled anti-mouse IgG antibody $(2.5 \mu \mathrm{g} / \mathrm{mL}$; goat anti-mouse IgG, Atto647N, Sigma Aldrich, Germany) were mixed in one batch and offered in a volume of $2 \mu \mathrm{L}$ to the beads from the opposite side. Both sides of the chamber were then sealed with $10 \mu \mathrm{L}$ mineral oil using the reservoirs. All components (capture probe-loaded microbeads, miRNAs and antibodies) were diluted with PBS-T buffer. The entire diffusion process was carried out in PBS-T.

\section{Diffusion approach II - miRNA diffusion in an antibody-saturated environment}

In contrast to the first approach, the capture probe-loaded microbeads (3-plex; see above) were added together with the anti-DNA:RNA hybrid antibody $(2.0 \mu \mathrm{g} / \mathrm{mL})$, and the secondary antibody $(2.5 \mu \mathrm{g} / \mathrm{mL})$ from one side of the microchamber (Fig. 2B). The respective miRNAs were offered to the microbead/antibody environment from the opposite site, and both sides were subsequently sealed with mineral oil. 


\section{Principle of antibody-based miRNA detection}

MiRNA-specific DNA capture probes were firstly immobilized on neutravidin-coated microbeads. The respective miRNA hybridizes with its capture probe, leading to the formation of a DNA:miRNA hybrid, which was then recognized by an anti-DNA:RNA hybrid antibody and a fluorescent secondary antibody (Fig. 2C).

The specificity of the anti-DNA:RNA hybrid antibody towards DNA:miRNA hybrids was shown in several studies. Complementary and thus perfect matched miRNAs to the capture probe lead to a perfect antibody binding (Fig. 3A), whereas single-base, multiple-base miRNA mismatches or non-complementary miRNA lead to impaired or no antibody binding (Fig. 3B) $[15,26]$.

\section{Fluorescence detection by VideoScan platform}

The fluorescence signals were monitored at room temperature $\left(24^{\circ} \mathrm{C} \pm 2{ }^{\circ} \mathrm{C}\right)$ and different time points ( $5 \mathrm{~min}, 40 \mathrm{~min}, 100 \mathrm{~min}, 160 \mathrm{~min}, 220 \mathrm{~min}, 280 \mathrm{~min}, 340 \mathrm{~min}$ and $24 \mathrm{~h}$ ) by the VideoScan platform, which automatically records 20 individual images per microchamber well. The platform is able to distinguish between different microbead populations due to their size and specific dye ratios of rhodamine $6 \mathrm{G}$ and coumarin 6 [17]. Microbeads of the same population have the same size, the same fluorescence dye ratio and a certain degree of carboxylation. Microbead-associated, and thus miRNA-specific, fluorescence signals (Atto647N) become visible as a red corona around the beads (Fig. 2D). 

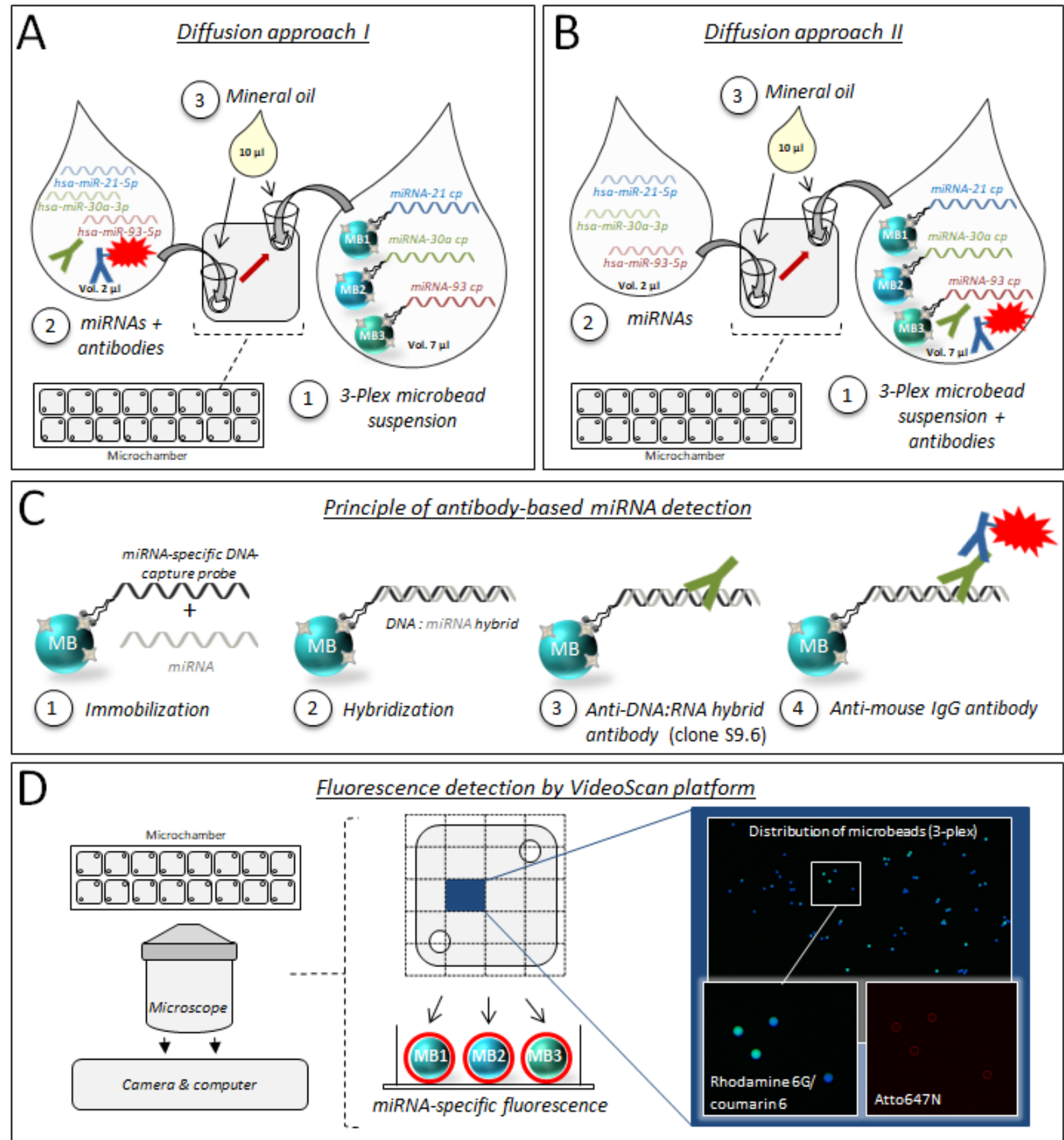

Fluorescence detection by VideoScan platform

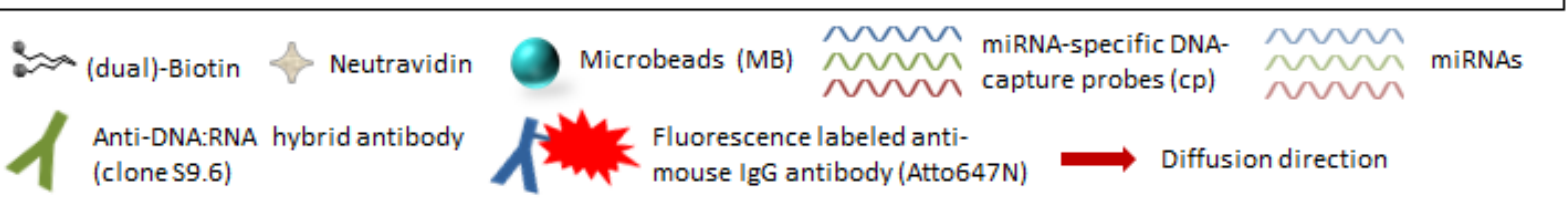

Figure 2. Multiplex microchamber diffusion assay workflow. Shown are two different experimental approaches: (A) "co-diffusion of miRNAs and antibodies" and (B) "miRNA diffusion in an antibody-saturated environment". In the first approach, a bulk mixed suspension of three microbead populations, each carrying a different specific DNA capture 
probe for miRNAs, was added from one side of the diffusion microchamber and the miRNAs (either as a mixture or individually) were applied together with the antibodies from the opposite side. In the second one, only the miRNAs (either as a mixture or individually) were added into the microchamber in which the microbeads and antibodies were already distributed. (C) Principle of miRNA detection with an anti-DNA:RNA hybrid antibody. (D) miRNA-specific fluorescence readout with the VideoScan platform. Shown are VideoScan false colour images of the distributed size/dye-encoded microbeads (3-plex) as well as miRNAspecific fluorescence signals (Atto647N) which become visible as a red corona around the beads.

\section{Characterization of DNA:miRNA hybrids}

The optimal temperature is important to minimize the contribution of mismatch nucleic acid species [27]. The melting temperatures of the DNA:miRNA hybrids on the surfaces of the microbeads were determined with the VideoScan heating and cooling unit (HCU) [17] and then evaluated with the MBmca package (v. 0.0.3-5) [20] using the R GUI /IDE RKWard version 0.7.1z+0.7.2+devel1 [28]. For this purpose, the DNA:miRNA hybrids were first formed on the microbeads by using synthetic DNA-fluorophor/miRNA-quencher pairs (Fig. 3C) (miRNA-21: 5'-Atto647N-TCA ACA TCA GTC TGA TAA GCT A-dual-Biotin-3'/ 5'UAG CUU AUC AGA CUG AUG UUG A-BHQ2-3'; miRNA-30a: 5'-Atto647N-GCT GCA ACA TCC GAC TGA AAG-dual-Biotin-3'/ 5'-CUU UCA GUC GGA UGU UUG CAG C-BHQ2-3'; miRNA-93: 5'-Atto647N-CTA CCT GCA CGA ACA GCA CTT TG-dual-Biotin-3' / 5'-CAA AGU GCU GUU CGU GCA GGU AG-BHQ2-3', Biomers.net, Germany). For the determination of the melting temperature, the temperature was then continuously increased from $30^{\circ} \mathrm{C}$ to $80^{\circ} \mathrm{C}(1$ ${ }^{\circ} \mathrm{C} /$ step) and the change in fluorescence intensity was recorded over time in PBS-T buffer. The melting temperatures were determined from the maximum of the first negative derivative (Table 1). Calculated melting temperatures and some other thermodynamic properties for the miRNAs used in this study are given in Table S1. 


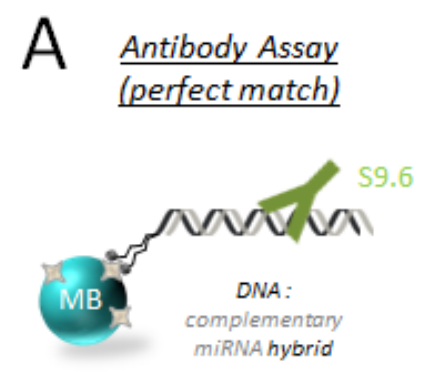

„perfect antibody binding

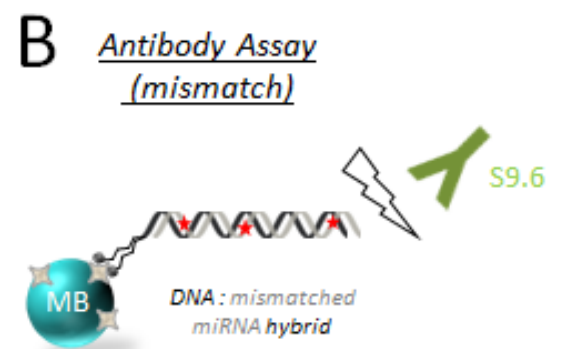

„impaired or no antibody binding
Kappel et al. 2015
Wang et al. 2015
C fret Assay

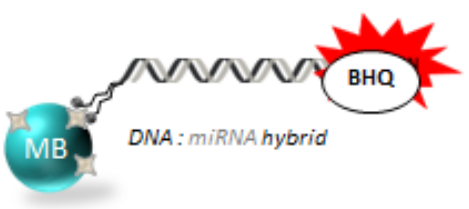

"direct hybridization via fluorophor/quencher pairing"

Figure 3. MiRNA detection methods. (A) Recognition of perfectly matched DNA:miRNA hybrids by the anti-DNA:RNA hybrid antibody clone S9.6. (B) Single-base or multiple-base mismatches between the miRNA-specific DNA capture probe and the miRNA lead to impaired or no antibody binding. The red stars indicate mismatches. (C) FRET-based direct hybridization of a fluorophore-labeled miRNA-specific DNA capture probe with a quencherlabeled miRNA. This FRET system was used for melting temperature determination.

Table 1: Determined melting temperatures of DNA:miRNA hybrids in the microbead-based FRET assay.

\begin{tabular}{lc}
\hline miRNA & $\begin{array}{c}\text { melting temperature } \\
\text { (experimental) } \\
T_{M}\left[{ }^{\circ} \mathrm{C}\right]\end{array}$ \\
\hline miR-21-5p & 54.8 \\
\hline miR-30a-3p & 56.5 \\
\hline miR-93-5p & 64.3 \\
\hline
\end{tabular}

\section{Data analysis}

\section{Data preprocessing, normalization and visualization}

The data were analyzed using analysis pipelines implemented in Python (3.+) to specifically analyze point pattern datasets. The raw VideoScan data (comma separated values) were reformatted to be read by the Python package pandas (v. 0.23.0). After extracting the fluorescence signal of population-specified microbeads, a threshold value using $99 \%$ quantile rule was calculated to detect microbeads with excessively high intensities at each 
time point. Those disproportionately fluorescent microbeads were assigned to the threshold value. Each microbead-specific fluorescence of each time point ( $5 \mathrm{~min}-24 \mathrm{~h}$ ) was normalized to the maximum fluorescence of the highest miRNA concentration (50 nM) of the $24 \mathrm{~h}$ measurement. The normalized signals were used to create the contour plots for distinct time points as well as miRNA concentrations using the Python package matplotlib (v. 2.2.2). To observe the number of positive microbeads at each time point, we used the 68-95-99.7 rule. Fluorescent signal intensities higher than mean $+3 \sigma$ of the PBS-T control over time were considered as positive. From that the means, given as percent, were presented as bar plots. The fluorescence intensities of those positive microbeads were plotted using boxplots.

\section{Dose-response curve fitting}

For the diffusion kinetics, the change over time (dose-response curve) of positive microbead percentages and the fluorescence intensities of those positive microbeads were fitted for each miRNA concentration by a four-parameter log-logistic function (Equation 1) [29],

$f(t)=c+\frac{d-c}{1+\exp (b *(\log (t)-\log (e)))}$

where $t$ is the time, $f(t)$ is the fluorescence intensity, $b$ is the hill slope, $c$ is the minimum intensity, $d$ is the maximum intensity, $e$ is the half maximal effective coefficient $\left(E C_{50}\right)$.

When making the dose-response curve fitting, we converted the $\mathrm{x}$-axis dose values to logarithmic scale $(\log 10(1 \mathrm{e} 2 *$ dose $)$. This will be helpful to plot the fitted curve into sigmoid shape, and this will make the relation between dose and response to be linear and therefore we can better choose the optimized dose values such as $E C_{50}$. The logarithm conversion values for time and concentration are shown in (Table S2).

For each of the two diffusion approaches, 3 biological replication experiments were conducted to verify the sensitivity of the detection system. Therefore, for each diffusion approach the mean $(n=3)$ and standard deviation was calculated, and the values were used to make the curve fitting for fluorescence intensity and the number of positive microbeads. 


\section{Quantification point estimation by Second Derivative Maximum method}

In mathematics, the first derivative can tell us the function $f(x)$ is increasing (positive) or decreasing (negative), and the second derivative tells us the change rate, so the second derivative maximum $(S D M)$ value could be interpreted as the inflection point with a maximum slope [30]. In this paper, with the use of the diff function from the Python sympy package, we calculated the SDM values for the time- and concentration-dependent increase curves of the number of positive microbeads and of the fluorescence intensities. We want to observe where the sigmoid curve gives the maximum turning point, and we can also compare the maximum turning points among different concentrations or time conditions.

\section{Complete spatial randomness (CSR)}

To test the randomness of the point pattern (spatial distribution) for each of the three microbead populations in a cavity of the microchamber, all cavities were judged against the hypothesis of complete spatial randomness (CSR). Therefore, we applied quadrat statistics to test individually the point pattern for all the microbead populations' distribution in each cavity of the microchamber of several assays (mean \pm standard deviation for diffusion approach I, $n=6$ and diffusion approach II, $n=6$ ). For $P<0.05$, we infer that the spatial distribution of our microbeads come not from a CSR process. This means that microbeads are not randomly distributed in the microchamber cavity. In addition to this we calculated the number of microbeads per area (unit) and the total number of microbeads per cavity of the chamber.

To investigate dependence between the points in a point pattern for each of the three microbead populations in the cavity, we estimated the cumulative distribution function $G$ $[31,32]$ of the nearest-neighbor distance for the typical point pattern $X$ (Equation 2):

$G(r)=P\{d(u, X \backslash\{u\}) \leq r \mid u \in X\}$

where $r$ is the distance argument, $u$ is an arbitrary location, and $d(\mathrm{u}, \mathrm{X} \backslash\{\mathrm{u}\})$ is the shortest

distance from $u$ to the point pattern $X$ excluding $u$ itself. For inferential purposes, the estimate of $G$ (empirical) is usually compared to the true value of $G$ (theoretical) for a completely random (Poisson) point process, if empirical $G(r)$ is bigger than theoretical $G(r)$. Thus the nearest-neighbour distances in the point pattern are shorter than for a Poisson 
process. This suggests a clustered pattern (where points tend to be close together), on the contrary it suggests a regular pattern (where points tend to avoid each other). For each of the three populations in the study, we calculated the experimental and theoretical $G(r)$ and found these two plots are very close, which suggests our microbeads are independently distributed in a poisson process pattern.

\section{Results and Discussion}

\section{Diffusion microchamber}

We developed a microchamber diffusion assay, which enables the multiplex detection of miRNAs on microbeads by antibodies in a small reaction environment. We used a setup, where the microbeads are randomly ordered ( $P$ value of CSR $>0.05$ ) (Table 2). Between 1,000 to 1,400 microbeads per population, which refer to $20-30$ microbeads per $\mathrm{mm}^{2}$, were used in a single microchamber (Table 2). This number led to an optimal and random distribution of the microbeads in the diffusion microchamber.

In this assay, the miRNAs to be detected diffuse through a field of DNA capture probeloaded microbeads, hybridize with their respective binding partners and form a DNA:miRNA hybrid. The hybrid is then detected by binding a primary antibody that specifically recognizes DNA:RNA hybrids, followed by binding a secondary fluorescencelabeled anti-mouse antibody that enables the detection of fluorescence signals by the VideoScan platform. A microbead-associated fluorescence signal can only be detected if the respective miRNA, the primary anti-DNA:RNA hybrid antibody and also the secondary, fluorescence-labeled antibody have found their particular target molecules.

The diffusion assay was performed in two different approaches. In the first approach, the miRNAs diffuse together with the primary and secondary antibodies through a field of microbeads loaded with capture probes (diffusion approach I - co-diffusion of miRNA and antibodies). In the second approach, however, only the miRNAs diffuse. Here, the two antibodies are already present in the microchamber along with the microbeads carrying the capture probes (diffusion approach II - diffusion of miRNAs in an antibody-saturated 
environment). We tested two approaches, in order to not lose any information e.g. regarding sensitivity or specificity.

Table 2. Test for random distribution of microbeads in the microchamber ( $p$ value of CSR), number of microbeads per area $\left(\mathrm{mm}^{2}\right)$ and total number of microbeads per cavity of the microchamber.

\begin{tabular}{clccc}
\hline population & $\begin{array}{c}\text { Microbead } \\
\text { pof }\end{array}$ & of CSR & $\begin{array}{c}\text { Number of } \\
\text { microbeads } \\
\text { per cavity }\end{array}$ & $\begin{array}{c}\text { Number of } \\
\text { microbeads } \\
\text { per area } \\
\left(\mathbf{m m}^{\mathbf{2}}\right)\end{array}$ \\
\hline Diffusion & MB1 (miR-21) & $0.40-0.75$ & $1,147 \pm 203$ & $24 \pm 4$ \\
approach & MB2 (miR-30a) & $0.23-0.64$ & $992 \pm 405$ & $20 \pm 8$ \\
I & MB3 (miR-93) & $0.41-0.84$ & $1,168 \pm 276$ & $24 \pm 6$ \\
\hline Diffusion & MB1 (miR-21) & $0.23-0.82$ & $1,208 \pm 242$ & $25 \pm 5$ \\
approach & MB2 (miR-30a) & $0.32-0.74$ & $1,085 \pm 432$ & $22 \pm 8$ \\
II & MB3 (miR-93) & $0.47-0.96$ & $1,393 \pm 438$ & $29 \pm 8$ \\
\hline
\end{tabular}

\section{Specificity of the microchamber diffusion assay}

To determine the specificity of our assay, we have carried out several experiments. The assay was first performed according to the principle of diffusion approach I (co-diffusion of miRNA and antibodies) with three selected miRNAs. In order to find out whether the complementary capture probes specifically bind their corresponding miRNAs, a 3-plex microbead mixture consisting of the populations MB1 with capture probe for miR-21 (MB1:miR-21_CP), MB2 with capture probe for miR-30a (MB2:miR-30a_CP) and MB3 with capture probe for miR-93 (MB3:miR-93a_CP) was inserted into the diffusion microchamber. The respective miRNA to be detected was added together with the antiDNA:RNA hybrid/secondary antibody mixture and the diffusion was recorded over time (Fig. 4). The diffusion of the three single miRNAs hsa-miR-21-5p (Fig. 4A), hsa-miR-30a-3p (Fig. 4B) or hsa-miR-93-5p (Fig. 4C) showed only fluorescence signals on the microbeads carrying the respective complementary capture probe. This indicated no unspecific binding of miRNAs (assay specificity of $96.7 \%$ ). Control experiments, in which only the primary and secondary antibody without any miRNA (PBS-T control) was added to the capture probe-loaded microbeads (3-plex) showed no unspecific signals. 
The miRNA binding to the capture probe-loaded microbeads could be observed in a timedependent manner. The spatial distribution and the intensity of the fluorescence signals increased over time, due to successively exposed miRNAs to the capture probe-loaded microbeads. In this approach the capture probe-loaded microbeads in front of the chamber were first occupied and subsequently saturated with the miRNAs and non-bonded miRNA molecules diffused further and could therefore subsequently bind to the microbeads with free binding sites.

The same procedure was carried out with the diffusion approach II (miRNA diffusion in an antibody saturated environment). The difference was in the experimental setup. In contrast to the approach mentioned above, the antibodies were placed in the chamber together with the capture probe-loaded microbeads and only the miRNAs diffuse. With regard to specificity, a higher false positive rate of $7.7 \%$ was found than for the diffusion approach I $(3.3 \%)$. 


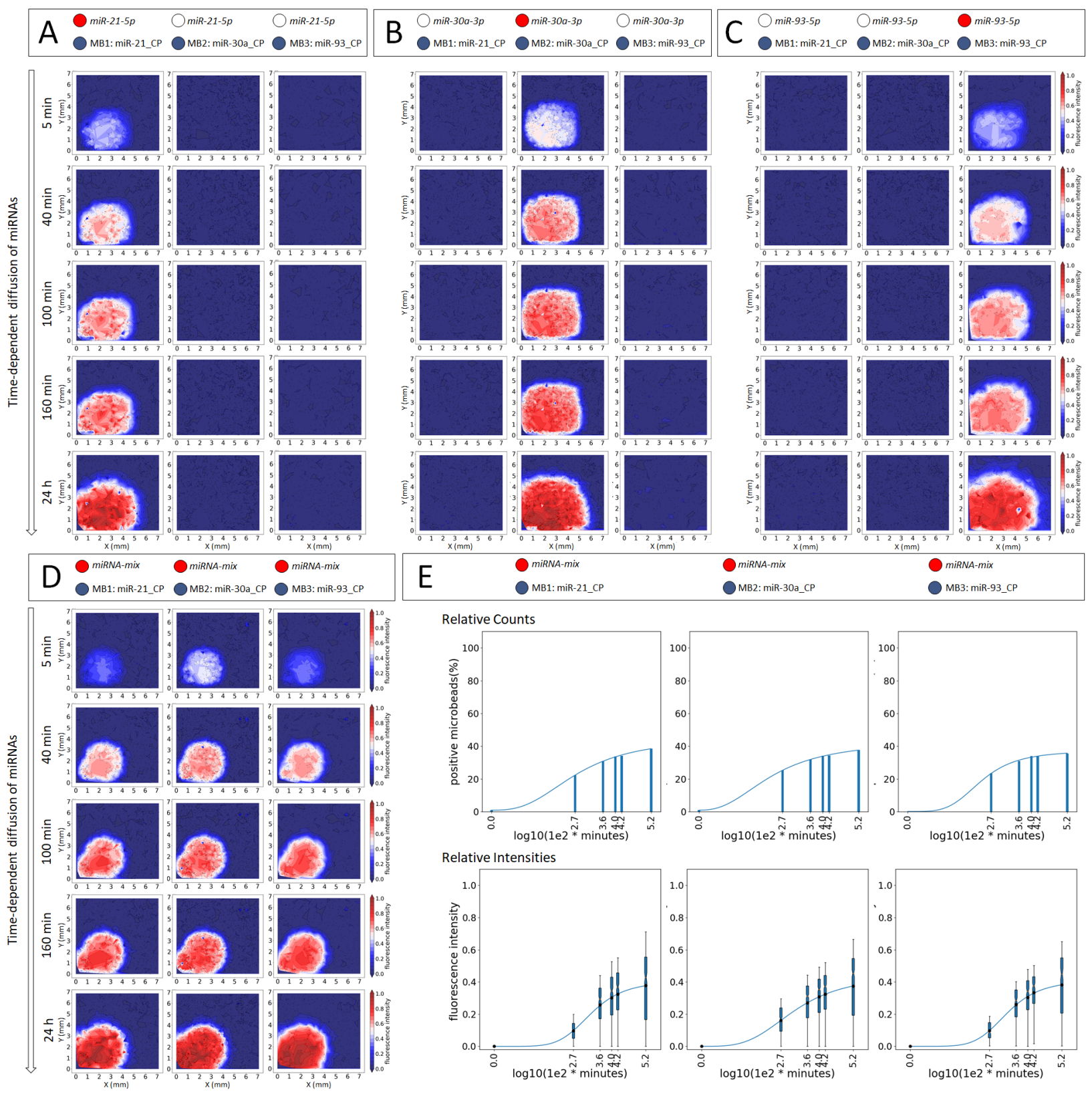

Figure 4. Multiplex diffusion-driven antibody-based detection of miRNAs in a microchamber. Time-dependent co-diffusion of (A) hsa-miR-21-5p (50 nM), (B) hsa-miR30a-3p (50 nM), (C) hsa-miR-93-5p (50 nM) and antibodies through a field of miRNA-specific capture probe-loaded microbeads (3-plex). The signal intensities of each microbead population are individually displayed as contour plots at different time points. (D) Codiffusion of the miRNA mixture (50 nM each hsa-miR-21-5p, hsa-miR-30a-3p and hsa-miR-93$5 p$ ) and antibodies through the 3-plex capture probe-loaded microbead field. (E) Shown are the percentages and box plots of mean fluorescence intensities of positive microbeads over time for the miRNA mixture diffusion. The contour plots, bar charts and box plots are exemplary for a representative experiment. All experiments were performed in triplicates. 
Circles indicate what has been added (blue = capture probe-loaded microbeads; red $=$ matching miRNA; unfilled = non-matching miRNA). $C P=$ capture probe, $M B=$ microbead.

\section{Multiplex detection of miRNAs}

In order to detect several miRNAs from a single sample (multiplex), we also tested the mixture of all three miRNAs (equal amounts of miRNAs) with both diffusion approaches. Co-diffusion of the miRNA mixture and antibodies against the capture probe-loaded microbeads (3-plex) is shown in Fig. 4D. Each miRNA of the mixture could be specifically detected in a time-dependent manner. Furthermore, diffusion kinetics were derived from the fluorescence data of the contour plots. These data are shown exemplarily for the miRNA mixture in Fig. 4E. First, the number of microbeads that became positive over the diffusion time was calculated and displayed as bar plots. All beads whose capture probes were already occupied with miRNA, i.e., which had a significantly higher fluorescence intensity than the PBS-T control, were considered as positive microbeads. From all those positive microbeads, we calculated the mean fluorescence intensities and displayed them for each time point as box plots. The number of microbeads that had successfully bound miRNA increased over time, and the same beads that initially bound only small amounts of miRNA bound more free miRNAs depending on time. This led ultimately to an increased fluorescence intensity over time, which could be fitted to a four-parameter log-logistic curve. Same results were observed for diffusion approach II.

For each diffusion approach (I and II), three independent experiments were carried out and respective diffusion kinetics (see Fig. 4E) were prepared. The mean diffusion kinetics (Fig. 5) were then obtained from the kinetics of the individual experiments. Figure 5A depicts the diffusion kinetics for the number of positive microbeads. The comparison of the kinetics showed that in diffusion approach I (upper panel) about $40 \%$ of the total microbeads became positive over time, whereas in diffusion approach II (lower panel) after $24 \mathrm{~h}$ almost $100 \%$ of the microchamber surface was covered with positive microbeads. However, the comparison of the mean fluorescence intensities of these positive microbeads did not show any major differences between the two diffusion approaches (Fig. 5B). Furthermore, in this 3-plex mixture, the capture probe-loaded microbeads had strong 
specificity to their respective miRNA. There was no fluorescence signal on those microbead populations where non-complementary capture probes were used for the miRNA detection (Fig. 5C-E).

Different parameters can be determined from the diffusion kinetics. In order to compare the performance of both diffusion approaches, the second derivative was used. This gives us a statistic about the inflection point in the sigmoid curve and indicates when a signal in the assay starts to become positive. The second derivative was determined for the number of microbeads becoming positive as well as the change of fluorescence intensities over time.
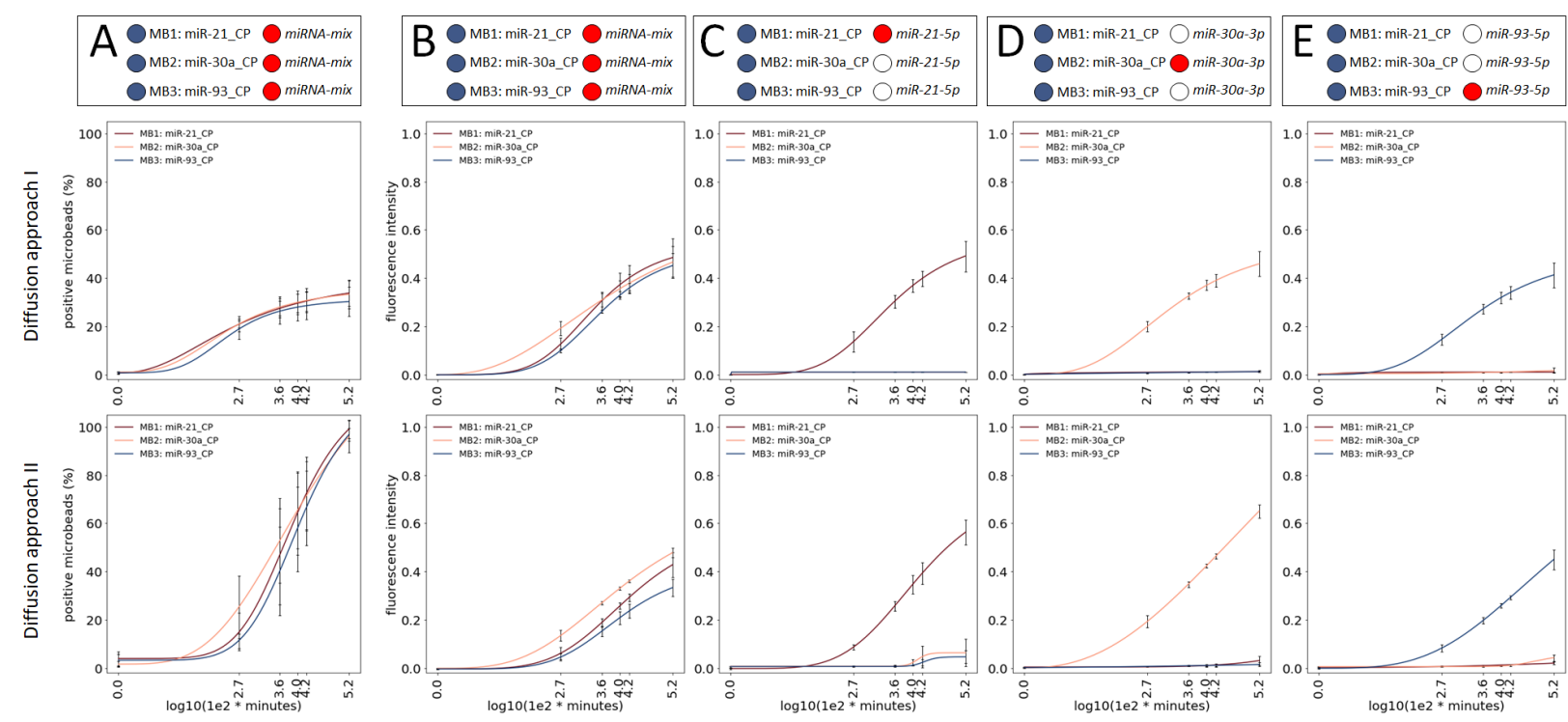

Figure 5. Diffusion kinetics for the multiplex antibody-based miRNA detection. Comparison of the kinetics of diffusion approach I and II regarding (A) number of positive microbeads in \% for the miRNA mixture and mean fluorescence intensities of positive microbeads for (B) miRNA mixture, (C) hsa-miR-21-5p (50 nM), (D) hsa-miR-30a-3p (50nM) and (E) hsa-miR-93-5p (50 nM). Shown are mean \pm standard deviation $(n=3)$ for each diffusion approach. Circles indicate what has been added (blue = capture probe-loaded microbeads; red = matching miRNA; unfilled = non-matching miRNA). $C P=$ capture probe, $M B$ = microbead. 


\section{Sensitivity and detection limit of the microchamber diffusion assay}

To evaluate the sensitivity of our diffusion-driven microchamber assay, we also investigated different miRNA concentrations ranging between $50 \mathrm{nM}$ down to $0.05 \mathrm{nM}$ as well as PBS-T control. The concentration-dependent miRNA detection was performed with both diffusion approaches (I and II) in the multiplex format.

\section{Diffusion approach I - co-diffusion of miRNAs and antibodies}

Since we were able to show that no unspecific binding or cross hybridization of the miRNAs used in this assay occurred, the co-diffusion approach was performed with seven miRNA dilutions in 3-plex.

Diffusion kinetics: The contour plots for each miRNA were displayed individually and are exemplified for miR-21-5p in Figure 6A. As can be seen, we could specifically differentiate between various miRNA concentrations, which lead to different fluorescence intensities due to the respective miRNA dilution. With increasing miRNA concentration, the number of microbeads with successfully bound miRNAs increased (Fig. 6B). By using smaller amounts of miRNA, the percentage of positive microbeads also decreased, as the smaller number of miRNA molecules limits the radius of propagation in the chamber. At a miR-21-5p concentration of $\geq 5 \mathrm{nM}$, approximately $10-15 \%$ of the total microbeads had already bound the miRNA and the two antibodies necessary for the detection of the hybrid within 5 minutes. After 40 minutes, about $30-40 \%$ of microbeads were positive. The percentage number of positive microbeads in this assay has not increased further with increasing diffusion time. Only the fluorescence intensity changed depending on time and miRNA concentration (Fig. 6C). This means that microbeads which had already bound small amounts of miRNA and were therefore considered positive, bound more miRNA molecules over time. This lead to an increase in fluorescence intensity of one and the same beads. 


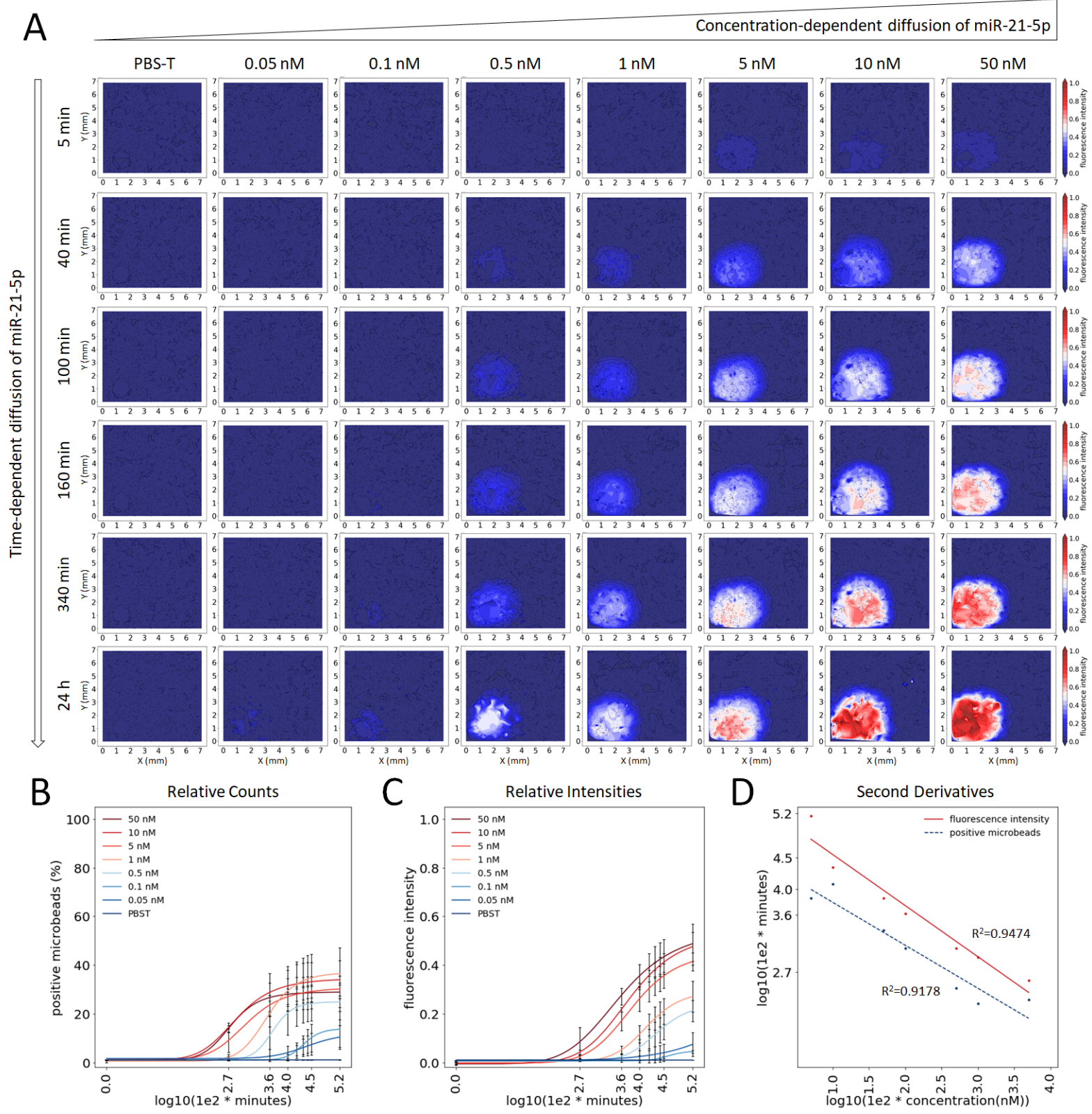

Figure 6. Diffusion approach I. (A) Contour plots of time- and concentration-dependent codiffusion of miRNAs and antibodies exemplified by miR-21-5p. (B) Diffusion kinetics of the positive microbeads as a function of the miRNA concentration. Shown is the percentage number of microbeads for miR-21-5p as mean \pm standard deviation $(n=3)$. Microbeads with fluorescence intensity above the buffer control (mean $+3 \sigma$ ) are considered as positive. (C) Diffusion kinetics of miR-21-5p concentration-dependent mean fluorescence intensities. (D) The second derivative maximum of the diffusion time in minutes for the number of positive microbeads and the mean fluorescence intensities are plotted against the miRNA concentration. The labeling of the $x$ - and $y$-axis are displayed as a logarithm of the concentration in $n M$, and the time point in minutes multiplied by 1 e2, respectively. 
In order to obtain a parameter for the sensitivity of this diffusion approach, the second derivative was derived from the concentration- and time-dependent fitted kinetics of the number of positive microbeads and the mean fluorescence intensities. The maximum of the respective second derivative ( $S D M$ ) was plotted as a function of the miRNA concentration (Fig. 6D). In this case, the SDM referred to the time point in the curve at which the slope is maximum and the curvature is zero. With a concentration of 10-50 nM miR-21-5p, positive microbeads with an average fluorescence signal above the threshold value could be detected already after 1.5 minutes. The use of smaller amounts of miRNA extended the time to signal ( $5 \mathrm{nM}$ after about $3 \mathrm{~min}, 1 \mathrm{nM}$ after $10 \mathrm{~min}, 0.5 \mathrm{nM}$ after $20 \mathrm{~min}, 0.1 \mathrm{nM}$ and $0.05 \mathrm{nM}$ after more than $100 \mathrm{~min}$ ). The $S D M$ of the mean fluorescence intensities of all positive microbeads was for $50 \mathrm{nM}$ at about $4 \mathrm{~min}, 10 \mathrm{nM}$ at $8 \mathrm{~min}, 5 \mathrm{nM}$ at $12 \mathrm{~min}, 1 \mathrm{nM}$ at $40 \mathrm{~min}, 0.5$ at $70 \mathrm{~min}, 0.1$ at $220 \mathrm{~min}$ and 0.05 at $1,400 \mathrm{~min}$.

Concentration-response curves: Furthermore, we derived concentration-response curves from the data by plotting the miRNA concentration against the fluorescence intensities for each time point and calculated the $E C_{50}$ values (Fig. S1-S3). The $E C_{50}$ refers to the concentration, at which in the sigmoid curve the half maximal response is achieved. An improvement of the $E C_{50}$ depending on the time can be observed for miR-21-5p (Fig. S1 A,C), miR-30a-3p (Fig. S2 A,C) and miR-93-5p (Fig. S3 A,C). But, after a diffusion time of 100 min no further improvement of the $E C_{50}$ can be achieved (Fig. S1-S3 C). By calculating the $S D M$ from the concentration-response curves, we see that, with the exception of miR-30a$3 p$, no higher sensitivity could be achieved due to continuing diffusion time (Fig. S1-S3 C).

\section{Diffusion approach II - miRNA diffusion in an antibody-saturated environment}

Diffusion kinetics: The contour plots show that with diffusion approach II, in which the antibodies are already present in the chamber, a concentration- and time-dependent miRNA detection like approach I was possible (Fig. 7A). However, independent of the miRNA concentration used, no microbeads had bound miR-21-5p after 5 minutes diffusion time (Fig. 7B). With concentrations $\geq 5 \mathrm{nM}, 30-40 \%$ of the microbeads were positive after about $40 \mathrm{~min}$. As the diffusion time increased, the percentage of positive microbeads increased even further up to $90-100 \%$, depending on the concentration. Within 24 hours, the entire chamber was completed with positive microbeads, at least at the higher miRNA 
concentrations. Lower miR-21-5p concentrations such as 1 or $0.5 \mathrm{nM}$ reached a maximum of $60 \%$ of the microbeads and with less than $0.5 \mathrm{nM}$ detection is unlikely. The fluorescence intensities of positive microbeads increased over time in a concentration-dependent manner (Fig. 7C). We also derived the $S D M$ from the fitted curves of Figure 7B and C. In contrast to diffusion approach I, with high miR-21-5p concentrations (10 and $50 \mathrm{nM}$ ) positive microbeads could only be detected after 25 and 20 minutes respectively (Fig. 7D). The time to signal increased by using lower miRNA concentrations (e.g. for $1 \mathrm{nM}$ after 30 $\mathrm{min}$, for $0.5 \mathrm{nM}$ after $50 \mathrm{~min}, 0.1 \mathrm{nM}$ and $0.05 \mathrm{nM}$ after $130 \mathrm{~min}$ and 1,400 min, respectively).

The $S D M$ of the mean fluorescence intensities of all positive microbeads was for $50 \mathrm{nM}$ at $12 \mathrm{~min}, 10 \mathrm{nM}$ at $15 \mathrm{~min}, 5 \mathrm{nM}$ at $25 \mathrm{~min}, 1 \mathrm{nM}$ at $75 \mathrm{~min}, 0.5$ at $110 \mathrm{~min}, 0.1$ at $140 \mathrm{~min}$ and 0.05 at $1,400 \mathrm{~min}$.

Concentration-response curves: We also derived concentration-response curves for miR-215p (Fig. S1 B,D), miR-30a-3p (Fig. S2 B,D) and miR-93-5p (Fig. S3 B,D) from the data of diffusion approach II and calculated $E C_{50}$ values from the fitted curves. After at least one hour diffusion time (between 40 and 100 minutes time points), there was no further change in the $E C_{50}$ values (Fig. S1-S3 D), i.e. with regard to this parameter, which is commonly used to determine affinity and thus sensitivity, a further incubation time did not lead to an improvement, which suggests that the miRNA detection had already been completed. Comparison of the time-dependent SDM values revealed the same (Fig. S1-S3 D). 


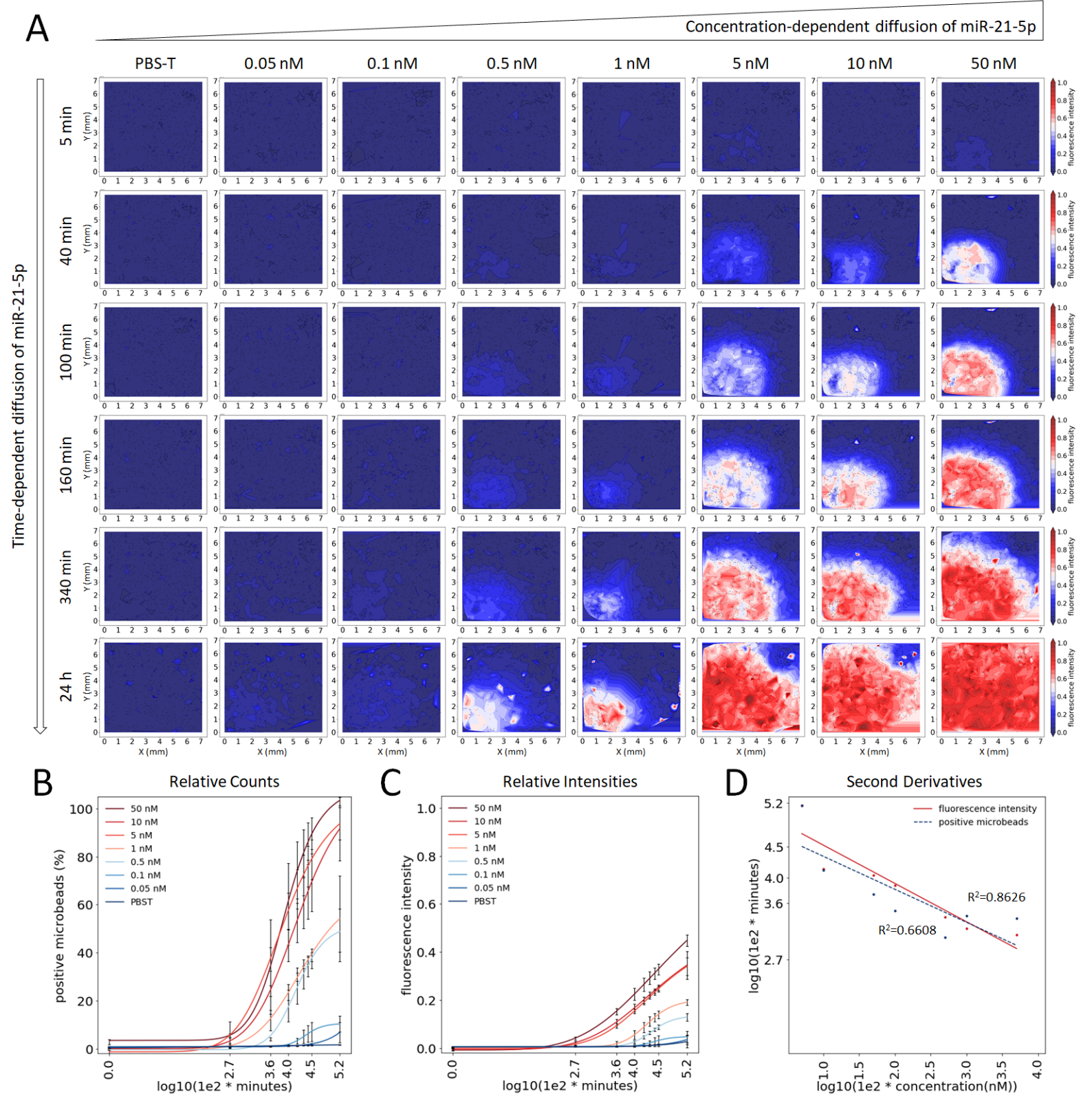

Figure 7. Diffusion approach II. (A) Contour plots of time- and concentration-dependent diffusion of miRNAs in an antibody saturated environment exemplified by miRNA-21. (B) Diffusion kinetics of the positive microbeads as a function of the miRNA concentration. Shown is the percentage number of microbeads for miR-21-5p as mean \pm standard deviation $(n=3)$. Microbeads with fluorescence intensity above the buffer control (mean $+3 \sigma)$ are considered as positive. (C) Diffusion kinetics of miR-21-5p concentration-dependent mean fluorescence intensities $(n=3)$. (D) The second derivative maximum of the diffusion time in minutes for the number of positive microbeads and the mean fluorescence intensities are plotted against the miRNA concentration. The labeling of the $x$-and $y$-axis are displayed as a logarithm of the concentration in $n M$, and the time point in minutes multiplied by 1e2, respectively. 


\section{Comparison of diffusion approach I and II}

When comparing the two diffusion approaches, it can be seen that each has advantages and disadvantages with regard to the parameters listed in Table 3.

Handling, specificity and multiplexing: The three investigated miRNAs could be detected specifically and in multiplex with both approaches. A rather better assay specificity (96.7 \% vs. $92.3 \%$ ) could be achieved with the first approach. The diffusion approach II offers a rather easier handling compared to I, because the antibodies do not have to be added to each individual miRNA dilution. By adding the antibodies together with the capture probeloaded microbeads in the chamber, assay errors might be reduced.

Signal-to-noise ratio: We observed detection differences depending on the experimental approach regarding the number of microbeads becoming positive over time and the overall fluorescence intensities. With the same amount of miRNA as in diffusion approach I, a larger detectable propagation radius was achieved in diffusion approach II (Fig. 6A, Fig. 7A). With diffusion approach I only $30-40 \%$ of all microbeads become positive for miR21-5p ( Fig. 6B), miR-30a-3p (Fig. S4 A) and miR-93-5p (Fig. S5 A), whereas with approach II for all three miRNAs 90-100 \% (Fig.7B, Fig. S4 D, Fig. S5 D) are achieved. Nevertheless, higher fluorescence intensities can be achieved with the first approach for all three miRNAs than with the second (Fig. 6B, Fig. 7B, Fig. S4 B,E, Fig. S5 B,E), which can be confirmed by better signal-to-noise ratios (Table S3).

Sensitivity: Although diffusion approach I seems to be more sensitive than approach II with regard to $E C_{50}$ and $S D M$ values, no significant differences can be determined, with the exception of miR-30a-3p for $E C_{50}$ or miR-93-5p for $S D M$ (Fig. S1- S3 C,D). The time to signal with respect to the number of positive microbeads and relative fluorescence intensities is faster with diffusion approach I than with diffusion approach II. Smaller amounts of miRNA can be detected earlier with approach I. 
Table 3. Comparison of both diffusion approaches. A plus sign indicates a positive evaluation and defines the ranking.

\begin{tabular}{lcc}
\hline & $\begin{array}{c}\text { Diffusion approach I } \\
\text { co-diffusion of } \\
\text { miRNA and antibodies }\end{array}$ & $\begin{array}{c}\text { Diffusion approach II } \\
\text { miRNA diffusion in an } \\
\text { antibody saturated- } \\
\text { environment }\end{array}$ \\
\hline Handling & ++ & +++ \\
Specificity & +++ & ++ \\
Multiplexing & +++ & +++ \\
Signal-to-noise ratio: & & +++ \\
Number of positive microbeads & + & ++ \\
Mean fluorescence intensity & +++ & ++ \\
Sensitivity: & & ++ \\
Time to signal & +++ & ++ \\
EC & +++ & +++ \\
SDM & & + \\
\hline
\end{tabular}

Since all conditions except the presence of the antibodies (co-diffusion or already present) were the same, this seems to be the limiting factor in approach I. Limiting at least in terms of the number of microbeads that became positive over time. This could be due to different diffusion rates of miRNA and antibodies. They differ in mass by the factor of about 21 [33], because a miRNA weighs about $7 \mathrm{kDa}$ and an IgG antibody approximately $150 \mathrm{kDa}$. The miRNAs were able to spread over the entire microchamber within $24 \mathrm{~h}$ by diffusion. This could be demonstrated by the approach II. In approach I, the antibodies, which are ultimately the signal generators, remained behind the miRNAs, i.e. the number of positive microbeads (in the maximum $40 \%$ ) refers to the propagation limit in which miRNA and antibodies still overlap. MiRNAs diffused further to the end of the chamber. They reached this end after about $24 \mathrm{~h}$. The antibodies were lagging behind due to their possible inertia, i.e. they might take longer than $24 \mathrm{~h}$ to diffuse further and bind free DNA:miRNA hybrids. The number of positive microbeads depends equally on how many miRNA molecules have bound and how well the DNA:miRNA hybrid is recognized by the primary and secondary antibody. A miRNA bound solely to the complementary capture probes of the microbead cannot be detected by VideoScan. The entire microbead complex with probe + miRNA + primary antibody + secondary antibody is always required for the detection. 
Another aspect is the gradient in the chamber itself. This applies to both approaches and to each dilution stage. There is always a gradient from a higher miRNA concentration at the entrance of the chamber to a lower concentration at the opposite end. This is due 1) to the fact that miRNA molecules bind to probes at the very beginning of the process and thus can diffuse less and less further until at the end all molecules are completely bound or an excess remains because all probes on the microbeads are already occupied. The latter could occur at too high concentrations. In the case of very small amounts of miRNA, the molecules are used up more quickly, i.e. bound to probes, and diffusion ends earlier. 2) It is due to the experimental dilution factor itself. Liquid in a volume of $7 \mu \mathrm{L}$ is presented in the chamber and the miRNA (with or without antibodies, depending on the approach) is dissolved in a volume of $2 \mu \mathrm{L}$. Mixing of both volumes results in gradual dilution.

Although the sensitivity of the presented miRNA detection method cannot compete with quantitative PCR, there are advantages in hybridizing miRNA with capture probe-loaded microbeads which are reached stepwise over time (diffusion assays), compared to those, which are simultaneously present (bulk mixed, reference assays) (Fig. S6-S8). In the reaction vessel, the hybridization of the miRNAs to the capture probes is non-directed and in the diffusion microchamber a directed propagation can be ensured. The diffusion chamber offers the possibility to count the microbeads, namely microbeads that become positive depending on time and miRNA concentration. It can also be clearly defined at which location a signal has to be emitted and at which not. False positive signals can therefore be excluded with the diffusion approach. This cannot be achieved in the reaction vessel, because all microbeads are mixed simultaneously and evenly.

It was shown that real-time DNA microarrays have several advantages over end-point measurements. Real-time systems have enhanced detection dynamic range and they are less dependent on probe saturation in the capturing spots and processing steps such as washing artifacts and microarray spot-to-spot variations [34].

The diffusion assay is suitable for multiplex detection of several miRNAs from one sample. Due to the tiny reaction cavity of the chamber, it is sample-saving and therefore applicable for small sample material. The presumed advantage is, that the miRNAs are successively 
exposed to the capture probe-loaded microbeads and that in this approach the capture probe-loaded microbeads in front are first saturated with the target molecules and nonbonded miRNAs diffuse further and can therefore subsequently bind to the microbeads with free binding sites further inside the chamber.

Briefly, the microbeads can be digitized into two groups: Firstly positive microbeads to which the miRNA has specifically bound (positive, 1) and secondly microbeads to which no miRNAs have bound within the elapsed time (negative, 0). Each microbead represents a measuring point that can be used to quantify the fluorescence intensity of the miRNAs (continuous variable) on the microbead. Since a certain number of miRNAs can bind specifically to each microbead until saturation, the signals increase over time. However, the signal increase is only noticeable at the microbeads, where the analyzed solution with the miRNA has already diffused. The other microbeads therefore have a negative signal. The diffusion of miRNAs is similar to a random walk.

So far, we are not using the available information of all the data types (discrete state (positive, negative) and continuous quantity) for the quantification of miRNAs. The diffusion assay provides the prerequisite for quantification. Quantification of miRNA is generally important because its expression (high/low) plays a role in many diseases, e.g. in the development of disease or its progress monitoring. The miRNAs could be quantified in our assay e.g. by the spreading radius, i.e. by the time of microbeads becoming positive. This radius depends on the miRNA concentration (for this purpose, an extra software tool would have to be developed).

Nevertheless, some limitations should be addressed. Diffusion is a slow process and therefore time-consuming. This fact, combined with the complex sample preparation, makes the application in a diagnostic laboratory currently challenging. Therefore, we intend to integrate this approach into a microfluidic platform to optimize handling and incubation time. For the test to be applicable, its sensitivity must be improved for routine diagnostics. Although it is possible to detect miRNAs in the nanomolar range, these concentrations are nevertheless above the picomolar levels found in body fluids such as plasma or serum. To solve this problem, we will try to validate buffer and temperature 
conditions, change the chamber geometry (e.g. microfluidics), use dendrimers or more intensely labeled antibodies and in addition antibodies against selected miRNAs, which can detect these miRNAs with a high sensitivity. We aim to gradually increase the multiplex level of the assay to allow the determination of as many miRNAs as possible from a single sample.

\section{Conclusion}

Our microbead-based microchamber diffusion assay is suitable for the antibody-based detection of miRNAs. By directly detecting a resulting DNA:miRNA hybrid with an antiDNA:RNA hybrid antibody, no labeling of the miRNA is necessary, which renders the assay universal and suitable for the detection of any relevant miRNA. The specificity of the assay can be guaranteed by using miRNA-specific complementary probes and the anti-DNA:RNA hybrid antibody only recognizes perfectly matching hybrids [15, 26, 35]. Furthermore, this assay allows the isothermal detection of miRNAs with both primary and secondary antibodies in a single reaction step without any amplification processes. To the best of our knowledge, this is the first assay that combines a directed diffusion of the analyte solution with a planar multiplex microbead array and antibody-based detection of miRNA and realtime monitoring.

The basic idea of the microchamber diffusion assay can be useful for other applications, e.g. for cell culture experiments in which specific target molecules diffuse past cells, receptor interaction studies where activators or inhibitors diffuse in a directed manner or generally for the detection of other nucleic acids or proteins.

\section{Acknowledgments}

We thank Philipp Müller, Alexander Böhm and Jörg Nitschke (BTU Cottbus-Senftenberg) for their contributions to the development of the assay. 


\section{Funding}

This work was funded by BMBF-Innovationsinitiative für die neuen Länder Unternehmen Region Wachstumskern Potenzial, miRMAK, 30WKP55B and in part by the Gesundheitscampus Brandenburg - digilog: Digitale und analoge Begleiter für eine alternde Bevölkerung initiative of the Brandenburg Ministry of Science, Research and Culture (MWFK).

\section{Conflict of interest}

\section{References}

1. Ardekani AM, Naeini MM (2010) The Role of MicroRNAs in Human Diseases. Avicenna J Med Biotechnol 2:161-79

2. Piletič K, Kunej T (2016) MicroRNA epigenetic signatures in human disease. Arch Toxicol 90:2405-2419. https://doi.org/10.1007/s00204-016-1815-7

3. Ikeda S, Kong SW, Lu J, Bisping E, Zhang H, Allen PD, Golub TR, Pieske B, Pu WT (2007) Altered microRNA expression in human heart disease. Physiol Genomics 31:367-373 . https://doi.org/10.1152/physiolgenomics.00144.2007

4. Kim SY, Morales CR, Gillette TG, Hill JA (2016) Epigenetic regulation in heart failure. Curr Opin Cardiol 31:255-65

5. Samanta S, Balasubramanian S, Rajasingh S, Patel U, Dhanasekaran A, Dawn B, Rajasingh J (2016) MicroRNA: A new therapeutic strategy for cardiovascular diseases. Trends Cardiovasc Med 26:407-19

6. George S, Rödiger S, Schröder C, Knaut M, Küpper J-H (2016) Development of multiplex PCR systems for expression profiling of human cardiomyocytes induced to proliferate by lentivirus transduction of upcyte genes. J Cell Biotechnol 2:35-55 . https://doi.org/10.3233/JCB-15025

7. Barrans JD, Allen PD, Stamatiou D, Dzau VJ, Liew CC (2002) Global gene expression profiling of end-stage dilated cardiomyopathy using a human cardiovascular-based cDNA microarray. Am J Pathol 160:2035-43

8. Towbin JA, Solaro RJ (2004) Genetics of dilated cardiomyopathy: more genes that kill. J Am Coll Cardiol 44:2041-3

9. Osterziel KJ, Perrot A (2005) Dilated cardiomyopathy: more genes means more phenotypes. Eur Heart J 26:751-4

10. Creemers EE, Tijsen AJ, Pinto YM (2012) Circulating microRNAs: novel biomarkers and extracellular communicators in cardiovascular disease? Circ Res 110:483-495 . https://doi.org/10.1161/CIRCRESAHA.111.247452

11. Steer CJ, Subramanian S (2012) Circulating microRNAs as biomarkers: a new frontier in diagnostics. Liver Transpl 18:265-9 
12. Pabinger S, Rödiger S, Kriegner A, Vierlinger K, Weinhäusel A (2014) A survey of tools for the analysis of quantitative PCR (qPCR) data. Biomol Detect Quantif 1:23-33 . https://doi.org/10.1016/j.bdq.2014.08.002

13. Kok MGM, de Ronde MWJ, Moerland PD, Ruijter JM, Creemers EE, Pinto-Sietsma SJ (2018) Small sample sizes in high-throughput miRNA screens: A common pitfall for the identification of miRNA biomarkers. Biomol Detect Quantif 15:1-5 . https://doi.org/10.1016/j.bdq.2017.11.002

14. Kalle E, Kubista M, Rensing C (2014) Multi-template polymerase chain reaction. Biomol Detect Quantif 2:11-29 . https://doi.org/10.1016/j.bdq.2014.11.002

15. Kappel A, Backes C, Huang Y, Zafari S, Leidinger P, Meder B, Schwarz H, Gumbrecht W, Meese E, Staehler CF, Keller A (2015) MicroRNA In Vitro Diagnostics Using Immunoassay Analyzers. Clin Chem 61:600-607 . https://doi.org/10.1373/clinchem.2014.232165

16. Geiss GK, Bumgarner RE, Birditt B, Dahl T, Dowidar N, Dunaway DL, Fell HP, Ferree S, George RD, Grogan T, James JJ, Maysuria M, Mitton JD, Oliveri P, Osborn JL, Peng T, Ratcliffe AL, Webster PJ, Davidson EH, Hood L, Dimitrov K (2008) Direct multiplexed measurement of gene expression with color-coded probe pairs. Nat Biotechnol 26:317325 . https://doi.org/10.1038/nbt1385

17. Rödiger S, Schierack P, Böhm A, Nitschke J, Berger I, Frömmel U, Schmidt C, Ruhland M, Schimke I, Roggenbuck D, Lehmann W, Schröder C (2013) A Highly Versatile Microscope Imaging Technology Platform for the Multiplex Real-Time Detection of Biomolecules and Autoimmune Antibodies. Adv Biochem Eng Biotechnol 133:35-74 . https://doi.org/10.1007/10_2011_132

18. Choi Y, Schmidt C, Tinnefeld P, Bald I, Rödiger S (2019) A new reporter design based on DNA origami nanostructures for quantification of short oligonucleotides using microbeads. Sci Rep 9:4769 . https://doi.org/10.1038/s41598-019-41136-x

19. Herrmann A, Rödiger SJ, Schmidt C, Schierack P, Schedler U (2019) Spatial Separation of Microbeads into Detection Levels by a Bioorthogonal Porous Hydrogel for SizeSelective Analysis and Increased Multiplexicity. Anal Chem. https://doi.org/10.1021/acs.analchem.9b01586

20. Rödiger S, Böhm A, Schimke I (2013) Surface Melting Curve Analysis with R. R J 5:3753

21. Spiess A-N, Deutschmann C, Burdukiewicz M, Himmelreich R, Klat K, Schierack P, Rödiger S (2015) Impact of Smoothing on Parameter Estimation in Quantitative DNA Amplification Experiments. Clin Chem 61:379-388 . https://doi.org/10.1373/clinchem.2014.230656

22. Chagovetz A, Blair S (2009) Real-time DNA microarrays: reality check. Biochem Soc Trans 37:471-475 . https://doi.org/10.1042/BST0370471

23. Rödiger S, Liebsch C, Schmidt C, Lehmann W, Resch-Genger U, Schedler U, Schierack P (2014) Nucleic acid detection based on the use of microbeads: a review. Microchim Acta 181:1151-1168 . https://doi.org/10.1007/s00604-014-1243-4

24. Rödiger S, Ruhland M, Schmidt C, Schröder C, Grossmann K, Böhm A, Nitschke J, Berger I, Schimke I, Schierack P (2011) Fluorescence Dye Adsorption Assay to Quantify Carboxyl Groups on the Surface of Poly(methyl methacrylate) Microbeads. Anal Chem 83:3379-3385 . https://doi.org/10.1021/ac103277s

25. Schmidt C, Schierack P, Gerber U, Schröder C, Choi Y, Bald I, Lehmann W, Rödiger S 
(2020) Streptavidin Homologues for Applications on Solid Surfaces at High Temperatures. Langmuir. https://doi.org/10.1021/acs.langmuir.9b02339

26. Wang M, Li B, Zhou Q, Yin H, Zhou Y, Ai S (2015) Label-free, Ultrasensitive and Electrochemical Immunosensing Platform for microRNA Detection Using AntiDNA:RNA Hybrid Antibody and Enzymatic Signal Amplification. Electrochimica Acta 165:130-135 . https://doi.org/10.1016/j.electacta.2015.03.011

27. Blair S, Williams L, Bishop J, Chagovetz A (2009) Microarray temperature optimization using hybridization kinetics. Methods Mol Biol Clifton NJ 529:171-196 . https://doi.org/10.1007/978-1-59745-538-1_12

28. Rödiger S, Friedrichsmeier T, Kapat P, Michalke M (2012) RKWard: a comprehensive graphical user interface and integrated development environment for statistical analysis with R. J Stat Softw 49:1-34 . https://doi.org/10.18637/jss.v049.i09

29. Ritz C, Baty F, Streibig JC, Gerhard D (2015) Dose-Response Analysis Using R. PLOS ONE 10:e0146021 . https://doi.org/10.1371/journal.pone.0146021

30. Rödiger S, Burdukiewicz M, Schierack P (2015) chipPCR: an R package to pre-process raw data of amplification curves. Bioinformatics 31:2900-2902 . https://doi.org/10.1093/bioinformatics/btv205

31. Cressie N (1992) STATISTICS FOR SPATIAL DATA. Terra Nova 4:613-617. https://doi.org/10.1111/j.1365-3121.1992.tb00605.x

32. Ripley BD (1991) Statistical inference for spatial processes. Cambridge university press

33. Qavi AJ, Kindt JT, Gleeson MA, Bailey RC (2011) Anti-DNA:RNA Antibodies and Silicon Photonic Microring Resonators: Increased Sensitivity for Multiplexed microRNA Detection. Anal Chem 83:5949-5956 . https://doi.org/10.1021/ac201340s

34. Hassibi A, Vikalo H, Riechmann JL, Hassibi B (2009) Real-time DNA microarray analysis. Nucleic Acids Res 37:e132-e132 . https://doi.org/10.1093/nar/gkp675

35. Boguslawski SJ, Smith DE, Michalak MA, Mickelson KE, Yehle CO, Patterson WL, Carrico RJ (1986) Characterization of monoclonal antibody to DNA.RNA and its application to immunodetection of hybrids. J Immunol Methods 89:123-30

36. Sugimoto N, Nakano S, Katoh M, Matsumura A, Nakamuta H, Ohmichi T, Yoneyama M, Sasaki M (1995) Thermodynamic parameters to predict stability of RNA/DNA hybrid duplexes. Biochemistry 34:11211-11216 


\section{Supplemental Information}

\section{Characterization of DNA:miRNA hybrids}

For the three miRNAs in this study, we also calculated the melting temperature and some other miRNA properties (at $37^{\circ} \mathrm{C}$ ) such as delta Gibbs free energy $\left(\Delta G^{\circ}\right)$, delta enthalpy $\left(\Delta H^{0}\right)$ and delta entropy $\left(\Delta S^{0}\right)$, see Table $S 1$. The $\Delta G^{0}$ of forming the DNA:miRNA hybrids was calculated using the nearest-neighbor method, and the nearest-neighbor parameters for DNA:RNA duplexes in $1 \mathrm{M} \mathrm{NaCl}$ buffer was from Sugimoto et al. [36]. The knowledge about the theoretical melting temperature was used during the assay design. In particular, an incubation temperature of $24^{\circ} \mathrm{C}$ was chosen as a starting point with stable DNA:miRNA hybrids.

Table S1. Thermodynamic parameters of miRNAs used in this study. Duplex formation of miRNA and DNA oligonucleotides in solution were calculated by nearest-neighbor analysis with the unified parameters in $1 \mathrm{M} \mathrm{NaCl}$ [36].

\begin{tabular}{|c|c|c|c|c|}
\hline miRNA & $\begin{array}{c}\text { melting } \\
\text { temperature } \\
\text { (calculated) } \\
\text { TM }\left[{ }^{\circ} \mathrm{C}\right] \\
\end{array}$ & $\begin{array}{c}\text { delta Gibbs } \\
\text { free energy } \\
\text { (calculated) } \\
\Delta G^{0}[\mathrm{~kJ} / \mathrm{mol}] \\
\end{array}$ & $\begin{array}{c}\text { delta } \\
\text { enthalpy } \\
\text { (calculated) } \\
\Delta H^{0}[\mathrm{~kJ} / \mathrm{mol}] \\
\end{array}$ & $\begin{array}{c}\text { delta } \\
\text { entropy } \\
(\text { calculated }) \\
\Delta S^{0}[\mathrm{~J} /(\mathrm{mol} \cdot \mathrm{K})] \\
\end{array}$ \\
\hline miR-21-5p & 50.05 & -94.98 & -721.74 & $-2,020.87$ \\
\hline miR-30a-3p & 51.05 & -102.93 & -835.96 & $-2,363.12$ \\
\hline miR-93-5p & 57.81 & -119.24 & -842.66 & $-2,332.16$ \\
\hline
\end{tabular}

\section{Dose-response curve fitting}

The logarithm conversion values for time and concentration are given in Table S2.

Table S2. Logarithmic time and concentration.

\begin{tabular}{cccc}
\hline time in minutes & $\mathbf{l o g} \mathbf{1 0}(\mathbf{1 e 2}$ minutes) & concentration in $\mathbf{~ M M ~}$ & $\begin{array}{c}\text { log10(1e2* concentr } \\
\text { ation(nM)) }\end{array}$ \\
\hline 5 & 2.7 & 0.00 & - \\
40 & 3.6 & 0.05 & 0.7 \\
100 & 4.0 & 0.1 & 1.0 \\
160 & 4.2 & 0.5 & 1.7 \\
220 & 4.3 & 1 & 2.0 \\
280 & 4.4 & 5 & 2.7 \\
340 & 4.4 & 10 & 3.0 \\
1,440 & 5.2 & 50 & 3.7 \\
\hline
\end{tabular}




\section{Sensitivity and detection limit of the microchamber diffusion assay}

Supporting information of this subsection include Fig. S1, Fig. S2, Fig. S3, Fig. S4, Fig. 5 and Table S3.
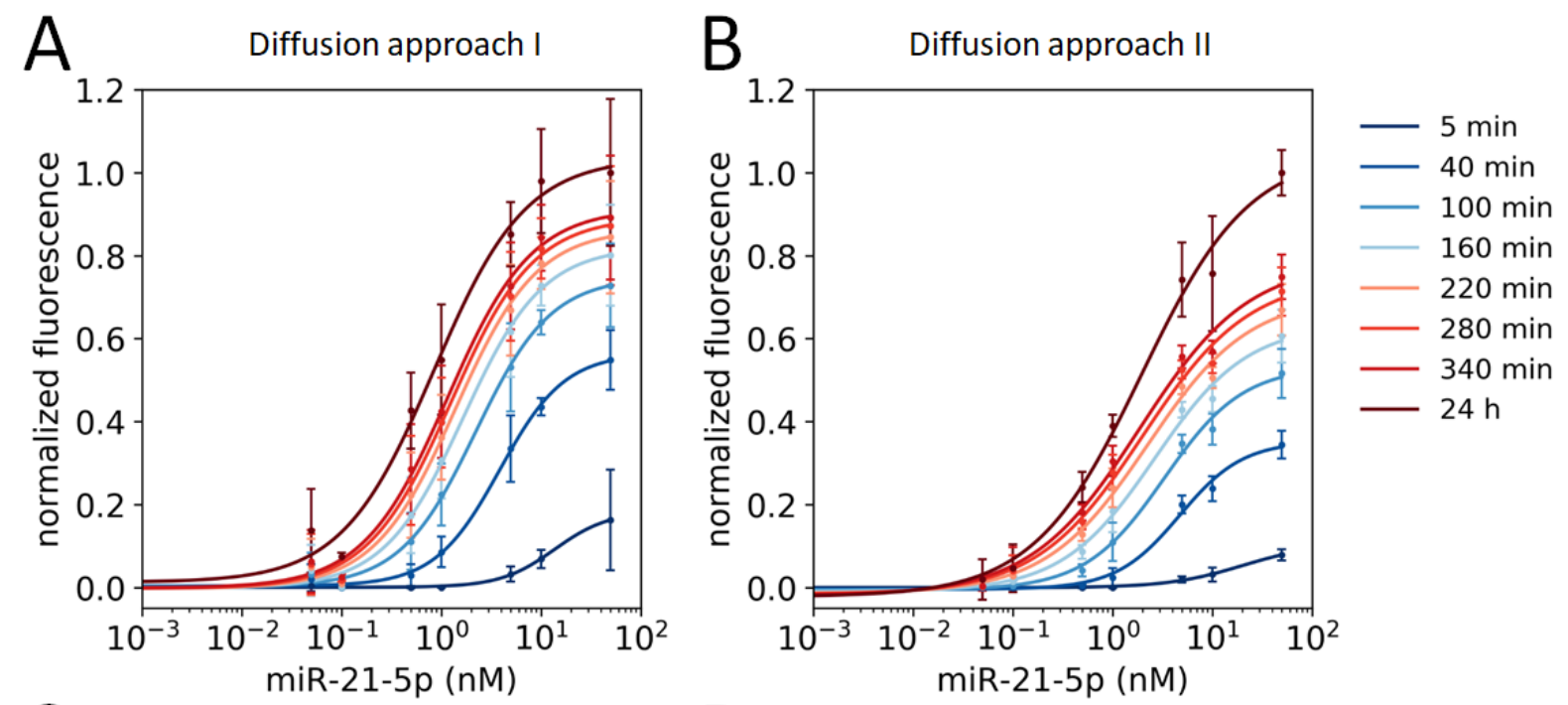

C
\begin{tabular}{|c|c|c|}
\hline Time & $\mathrm{EC}_{50}(\mathrm{nM})$ & $\mathrm{SDM}(\mathrm{nM})$ \\
\hline $5 \mathrm{~min}$ & $8.93 \pm 3.99$ & $5.17 \pm 2.83$ \\
\hline $40 \mathrm{~min}$ & $4.11 \pm 0.73^{\mathrm{ns}}$ & $1.83 \pm 1.06^{\mathrm{ns}}$ \\
\hline $100 \mathrm{~min}$ & $2.41 \pm 0.78^{\mathrm{ns}}$ & $0.77 \pm 0.39^{\mathrm{ns}}$ \\
\hline $160 \mathrm{~min}$ & $1.83 \pm 0.65^{\mathrm{ns}}$ & $0.54 \pm 0.28^{\mathrm{ns}}$ \\
\hline $220 \mathrm{~min}$ & $1.53 \pm 0.55^{\mathrm{ns}}$ & $0.44 \pm 0.24^{\mathrm{ns}}$ \\
\hline $280 \mathrm{~min}$ & $1.36 \pm 0.49^{\mathrm{ns}}$ & $0.38 \pm 0.21^{\mathrm{ns}}$ \\
\hline $340 \mathrm{~min}$ & $1.25 \pm 0.45^{\mathrm{ns}}$ & $0.34 \pm 0.19^{\mathrm{ns}}$ \\
\hline $24 \mathrm{~h}$ & $0.88 \pm 0.26^{\mathrm{ns}}$ & $0.21 \pm 0.12^{\mathrm{ns}}$ \\
\hline
\end{tabular}

$\mathrm{D}$

\begin{tabular}{|c|c|c|}
\hline Time & $\mathrm{EC}_{50}(\mathrm{nM})$ & SDM (nM) \\
\hline $5 \mathrm{~min}$ & $36.25 \pm 19.44^{(n s)}$ & $22.07 \pm 19.93^{\text {(ns) }}$ \\
\hline $40 \mathrm{~min}$ & $4.85 \pm 0.47^{*(n s)}$ & $1.69 \pm 0.30^{\mathrm{ns}(\mathrm{ns})}$ \\
\hline $100 \mathrm{~min}$ & $3.41 \pm 0.45^{*}(n s)$ & $0.91 \pm 0.19^{*}(\mathrm{~ns})$ \\
\hline $160 \mathrm{~min}$ & $2.83 \pm 0.64^{\mathrm{ns}(\mathrm{ns})}$ & $0.60 \pm 0.15^{\mathrm{ns}(\mathrm{ns})}$ \\
\hline $220 \mathrm{~min}$ & $2.58 \pm 0.85^{\mathrm{ns}(\mathrm{ns})}$ & $0.42 \pm 0.09^{n s(n s)}$ \\
\hline $280 \mathrm{~min}$ & $2.50 \pm 1.06^{\mathrm{ns}(\mathrm{ns})}$ & $0.35 \pm 0.08^{n s(n s)}$ \\
\hline $340 \mathrm{~min}$ & $2.49 \pm 1.24^{\mathrm{ns}(\mathrm{ns})}$ & $0.31 \pm 0.07^{n s(n s)}$ \\
\hline $24 \mathrm{~h}$ & $3.12 \pm 1.85^{\mathrm{ns}(\mathrm{ns})}$ & $0.33 \pm 0.13^{\mathrm{ns}(\mathrm{ns})}$ \\
\hline
\end{tabular}

Figure S1. Hsa-miR-21-5p. Concentration-response curves (mean \pm standard deviation) for diffusion approach (A) I and (B) II at different time points $(n=3)$. The data were normalized to the maximum of the 24 hour measurement. Shown are $E C_{50}$ and $S D M$ values for hsa-miR-21$5 p$ of diffusion approach (C) I and (D) II. The $E C_{50}$ and SDM values were calculated for each individual experiment and displayed as mean \pm standard deviation ( $n=3$, two-sided $t$-test, $\leq 0.05^{*}, \leq 0.01^{* *}, \leq 0.001^{* * *}$, nonsignificant ( $\left.n s\right)$ ). The significance levels between diffusion approach I and II are given in brackets. Significance levels between successive points in time within a diffusion assay are given without brackets. 

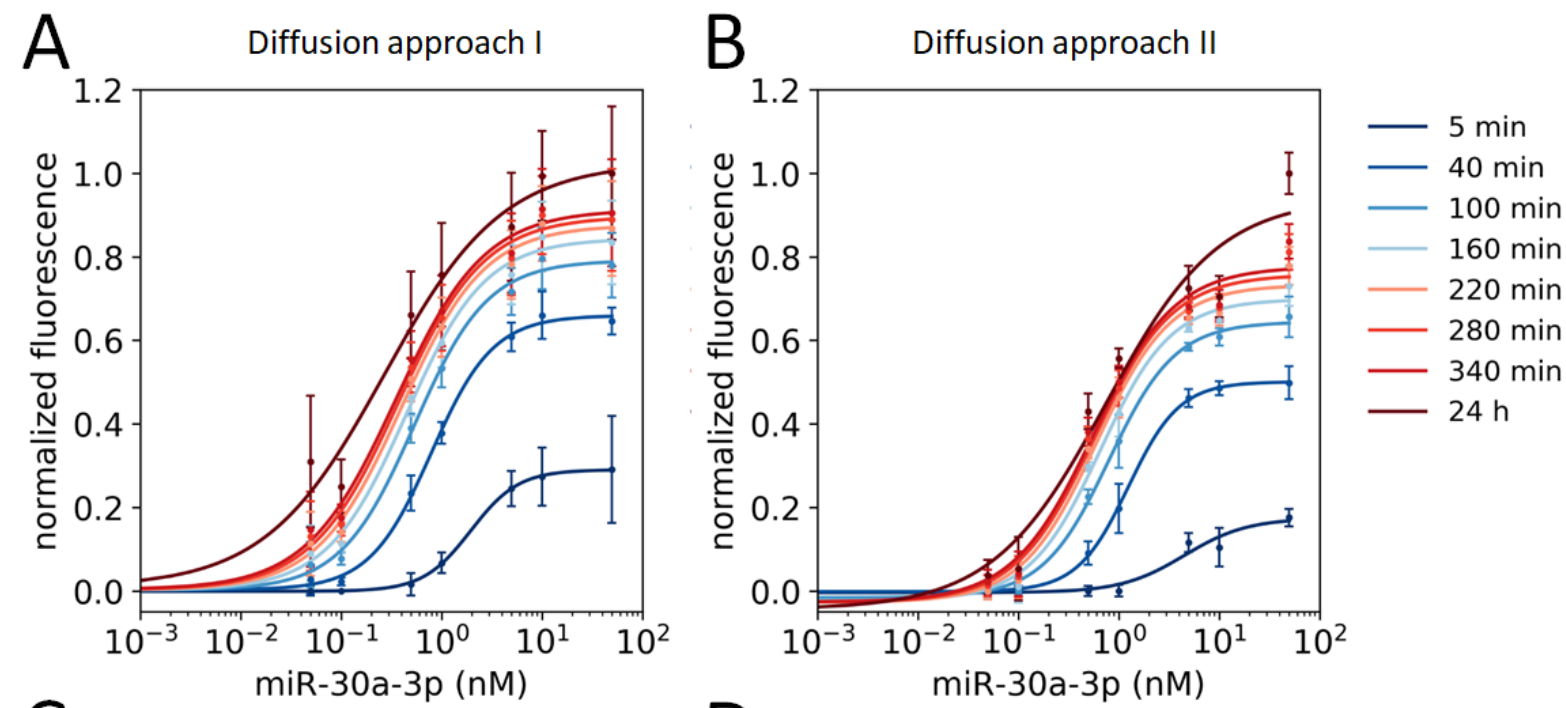

C
\begin{tabular}{|c|c|c|}
\hline Time & $\mathrm{EC}_{50}(\mathrm{nM})$ & $\mathrm{SDM}(\mathrm{nM})$ \\
\hline $5 \mathrm{~min}$ & $3.09 \pm 2.58$ & $1.25 \pm 0.56$ \\
\hline $40 \mathrm{~min}$ & $0.81 \pm 0.04^{\mathrm{ns}}$ & $0.31 \pm 0.07^{*}$ \\
\hline $100 \mathrm{~min}$ & $0.54 \pm 0.05^{*}$ & $0.16 \pm 0.01^{*}$ \\
\hline $160 \mathrm{~min}$ & $0.45 \pm 0.04^{\mathrm{ns}}$ & $0.12 \pm 0.00^{* *}$ \\
\hline $220 \mathrm{~min}$ & $0.40 \pm 0.03^{\mathrm{ns}}$ & $0.10 \pm 0.00^{* *}$ \\
\hline $280 \mathrm{~min}$ & $0.37 \pm 0.03^{\mathrm{ns}}$ & $0.08 \pm 0.00^{*}$ \\
\hline $340 \mathrm{~min}$ & $0.35 \pm 0.03^{\mathrm{ns}}$ & $0.07 \pm 0.01^{\mathrm{ns}}$ \\
\hline $24 \mathrm{~h}$ & $0.26 \pm 0.02^{*}$ & $0.03 \pm 0.02^{*}$ \\
\hline
\end{tabular}

\begin{tabular}{|c|c|c|}
\hline Time & $\mathrm{EC}_{50}(\mathrm{nM})$ & SDM (nM) \\
\hline $5 \mathrm{~min}$ & $2.88 \pm 0.96^{(n s)}$ & $1.31 \pm 0.21^{(\mathrm{ns})}$ \\
\hline $40 \mathrm{~min}$ & $1.21 \pm 0.18^{\mathrm{ns}(*)}$ & $0.54 \pm 0.13^{*(n s)}$ \\
\hline $100 \mathrm{~min}$ & $0.81 \pm 0.10^{\mathrm{ns}(*)}$ & $0.27 \pm 0.07^{*}(\mathrm{~ns})$ \\
\hline $160 \mathrm{~min}$ & $0.67 \pm 0.07^{\mathrm{ns}(*)}$ & $0.20 \pm 0.05^{\mathrm{ns}(\mathrm{ns})}$ \\
\hline $220 \mathrm{~min}$ & $0.62 \pm 0.07^{n s(*)}$ & $0.16 \pm 0.05^{\mathrm{ns}(\mathrm{ns})}$ \\
\hline $280 \mathrm{~min}$ & $0.60 \pm 0.06^{\mathrm{ns}(* *)}$ & $0.15 \pm 0.04^{\text {ns(ns) }}$ \\
\hline $340 \mathrm{~min}$ & $0.59 \pm 0.06^{\mathrm{ns}(* *)}$ & $0.14 \pm 0.04^{\text {ns(ns) }}$ \\
\hline $24 \mathrm{~h}$ & $0.81 \pm 0.16^{\mathrm{ns}(* *)}$ & $0.37 \pm 0.40^{\text {ns(ns) }}$ \\
\hline
\end{tabular}

Figure S2. Hsa-miR-30a-3p. Concentration-response curves (mean \pm standard deviation) for diffusion approach (A) I and (B) II at different time points $(n=3)$. The data were normalized to the maximum of the 24 hour measurement. Shown are $E C_{50}$ and SDM values for hsa-miR-30a$3 p$ of diffusion approach (C) I and (D) II. The $E C_{50}$ and SDM values were calculated for each individual experiment and displayed as mean \pm standard deviation ( $n=3$, two-sided $t$-test, $\leq 0.05^{*}, \leq 0.01^{* *}, \leq 0.001^{* * *}$, nonsignificant (ns)). The significance levels between diffusion approach I and II are given in brackets. Significance levels between successive points in time within a diffusion assay are given without brackets. 
Diffusion approach I

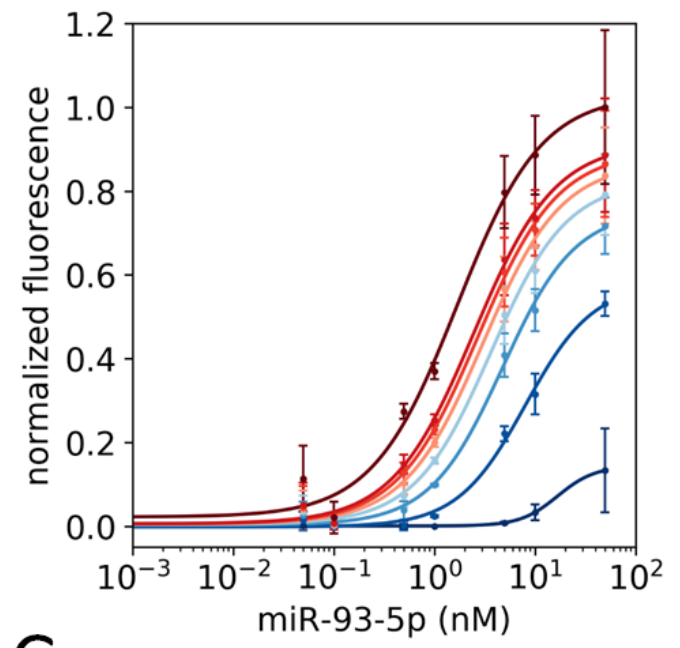

C
\begin{tabular}{|c|c|c|}
\hline Time & $\mathrm{EC}_{50}(\mathrm{nM})$ & $\mathrm{SDM}(\mathrm{nM})$ \\
\hline $5 \mathrm{~min}$ & $24.10 \pm 18.75$ & $21.92 \pm 20.10$ \\
\hline $40 \mathrm{~min}$ & $9.90 \pm 3.66^{\mathrm{ns}}$ & $3.02 \pm 0.10^{\mathrm{ns}}$ \\
\hline $100 \mathrm{~min}$ & $5.42 \pm 1.39^{\mathrm{ns}}$ & $1.81 \pm 0.67^{\mathrm{ns}}$ \\
\hline $160 \mathrm{~min}$ & $4.09 \pm 1.16^{\mathrm{ns}}$ & $1.11 \pm 0.23^{\mathrm{ns}}$ \\
\hline $220 \mathrm{~min}$ & $3.39 \pm 1.03^{\mathrm{ns}}$ & $0.86 \pm 0.14^{\mathrm{ns}}$ \\
\hline $280 \mathrm{~min}$ & $3.00 \pm 0.93^{\mathrm{ns}}$ & $0.75 \pm 0.09^{\mathrm{ns}}$ \\
\hline $340 \mathrm{~min}$ & $2.74 \pm 0.87^{\mathrm{ns}}$ & $0.67 \pm 0.07^{\mathrm{ns}}$ \\
\hline $24 \mathrm{~h}$ & $1.91 \pm 0.68^{\mathrm{ns}}$ & $0.43 \pm 0.03^{*}$ \\
\hline
\end{tabular}

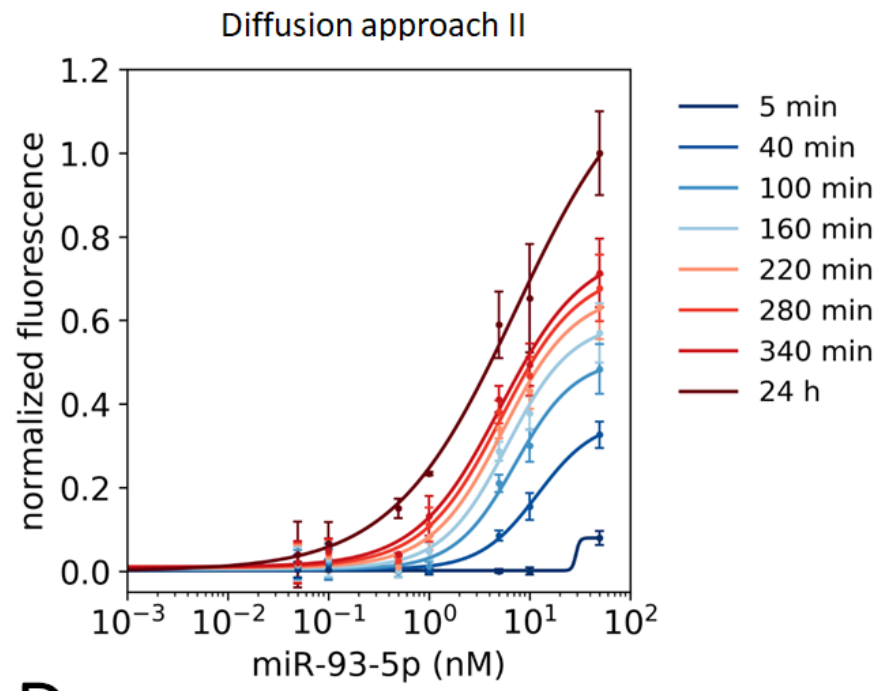

D

\begin{tabular}{|c|c|c|}
\hline Time & $\mathrm{EC}_{50}(\mathrm{nM})$ & SDM (nM) \\
\hline $5 \mathrm{~min}$ & $>50^{(\mathrm{ns})}$ & $>50^{(\mathrm{ns})}$ \\
\hline $40 \mathrm{~min}$ & $14.89 \pm 6.99^{* *(n s)}$ & $6.22 \pm 3.02^{* * *(n s)}$ \\
\hline $100 \mathrm{~min}$ & $8.03 \pm 1.65^{\text {ns(ns) }}$ & $2.75 \pm 0.58^{\text {ns(ns) }}$ \\
\hline $160 \mathrm{~min}$ & $6.62 \pm 1.24^{\mathrm{ns}(\mathrm{ns})}$ & $2.07 \pm 0.40^{n s(*)}$ \\
\hline $220 \mathrm{~min}$ & $6.17 \pm 1.63^{\text {ns(ns) }}$ & $1.68 \pm 0.34^{\mathrm{ns}(*)}$ \\
\hline $280 \mathrm{~min}$ & $6.12 \pm 2.15^{\mathrm{ns}(\mathrm{ns})}$ & $.49 \pm 0.39^{n s(*)}$ \\
\hline $340 \mathrm{~min}$ & $6.27 \pm 2.71^{\text {ns(ns) }}$ & $1.35 \pm 0.40^{\mathrm{ns}(*)}$ \\
\hline $24 \mathrm{~h}$ & $5.69 \pm 1.54^{\mathrm{ns}(\mathrm{ns})}$ & $1.09 \pm 0.32^{n 5^{(*)}}$ \\
\hline
\end{tabular}

Figure S3. Hsa-miR-93-5p. Concentration-response curves (mean \pm standard deviation) for diffusion approach (A) I and (B) II at different time points $(n=3)$. The data were normalized to the maximum of the 24 hour measurement. Shown are $E C_{50}$ and $S D M$ values for hsa-miR-93-5p of diffusion approach (C) I and (D) II. The $E C_{50}$ and SDM values were calculated for each individual experiment and displayed as mean \pm standard deviation ( $n=3$, two-sided t-test, $\leq 0.05^{*}, \leq 0.01^{* *}, \leq 0.001^{* * *}$, nonsignificant (ns)). The significance levels between diffusion approach I and II are given in brackets. Significance levels between successive points in time within a diffusion assay are given without brackets. 

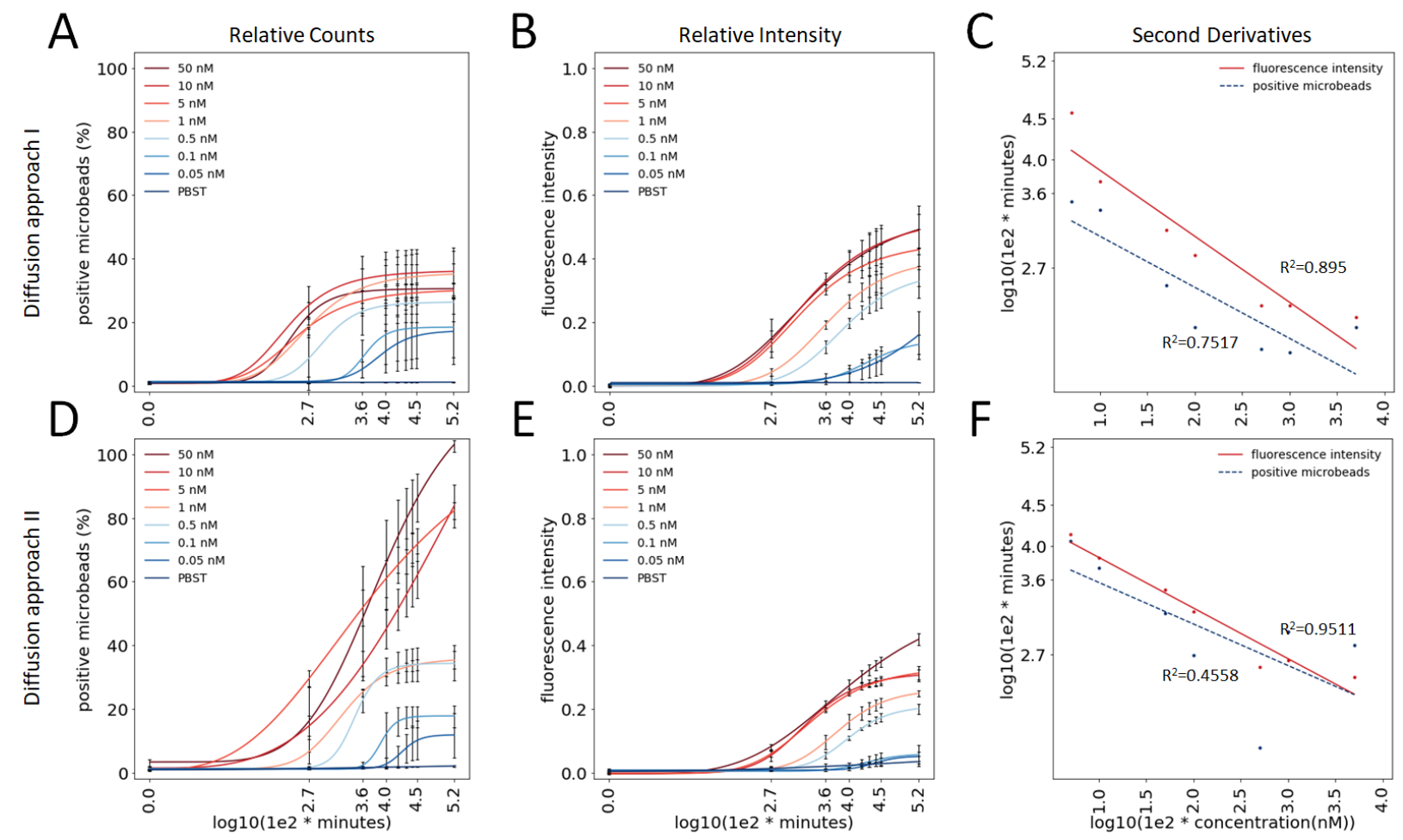

Figure S4. Comparison of the diffusion kinetics of hsa-miR-30a-3p for diffusion approach I and II. (A/D) Percentage change in the number of positive microbeads as a function of the miRNA concentration. (B/E) Change of mean fluorescence intensities of positive microbeads at different miRNA concentrations. Shown are mean \pm standard deviation of $n=3$ each. $(C / F)$ The second derivative maximum of the diffusion time in minutes for the number of positive microbeads and the mean fluorescence intensities are plotted against the miRNA concentration. 

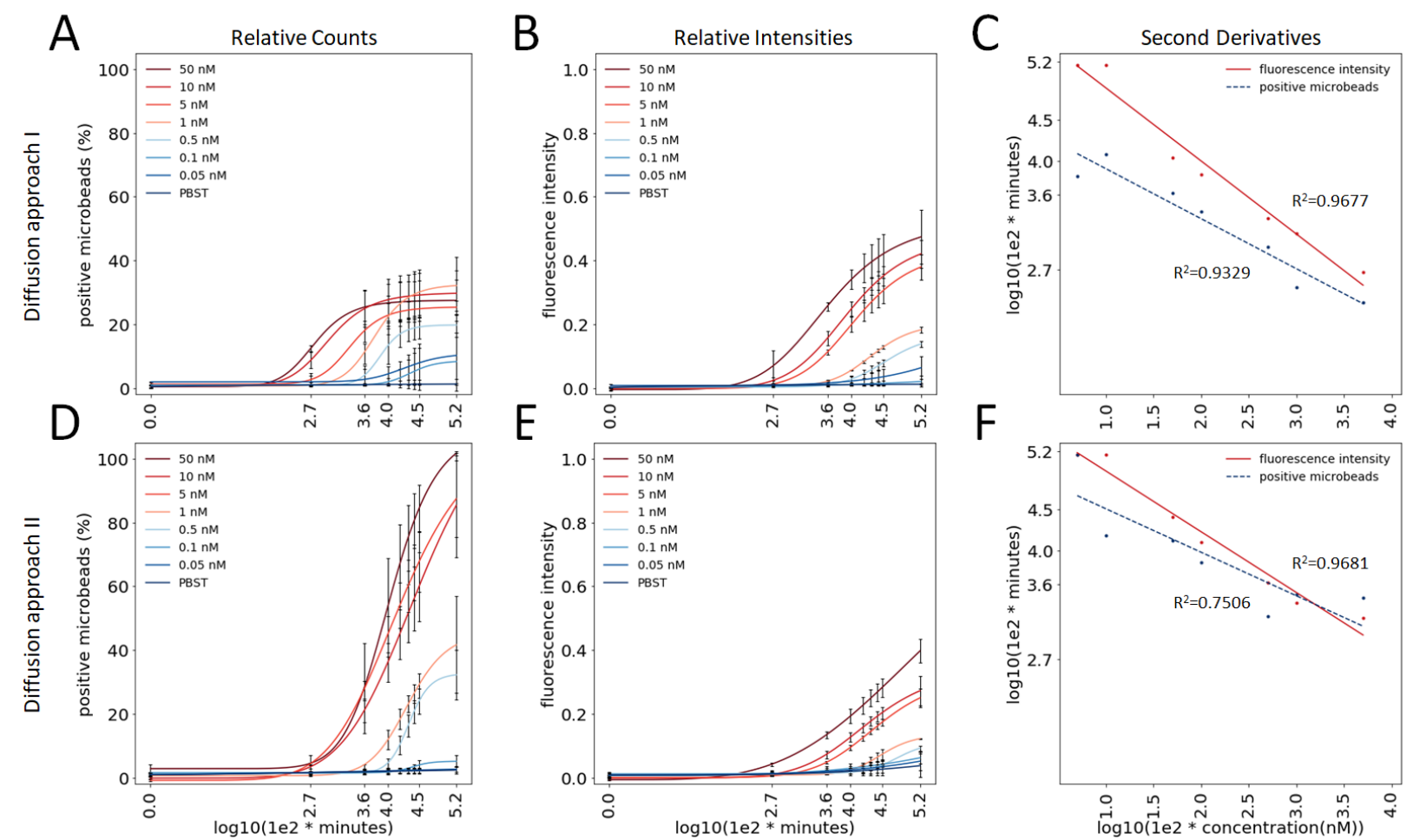

Figure S5. Comparison of the diffusion kinetics of hsa-miR-93-5p for diffusion approach I and II. $(A / D)$ Percentage change in the number of positive microbeads as a function of the miRNA concentration. ( $B / E)$ Change of mean fluorescence intensities of positive microbeads at different miRNA concentrations. Shown are mean \pm standard deviation of $n=3$ each. $(C / F)$ The second derivative maximum of the diffusion time in minutes for the number of positive microbeads and the mean fluorescence intensities are plotted against the miRNA concentration.

Table S3. Signal-to-noise ratios. The signal-to-noise ratios (SNR) were calculated for the diffusion assay I (DAI), the diffusion assay II (DAII), the reference assay adapted to the diffusion assay (Ref. DA adapted) and the standard reference assay (Ref. Standard) by the equation $S N R=10 \log _{10}\left(\frac{\text { signal }}{\text { noise }}\right)$, where signal is the fluorescence intensity of the tested miRNAs at different concentrations and time points, and noise the fluorescence intensity of the respective $P B S T$ control. Higher $d B$ values indicate better signal-to-noise ratios. 


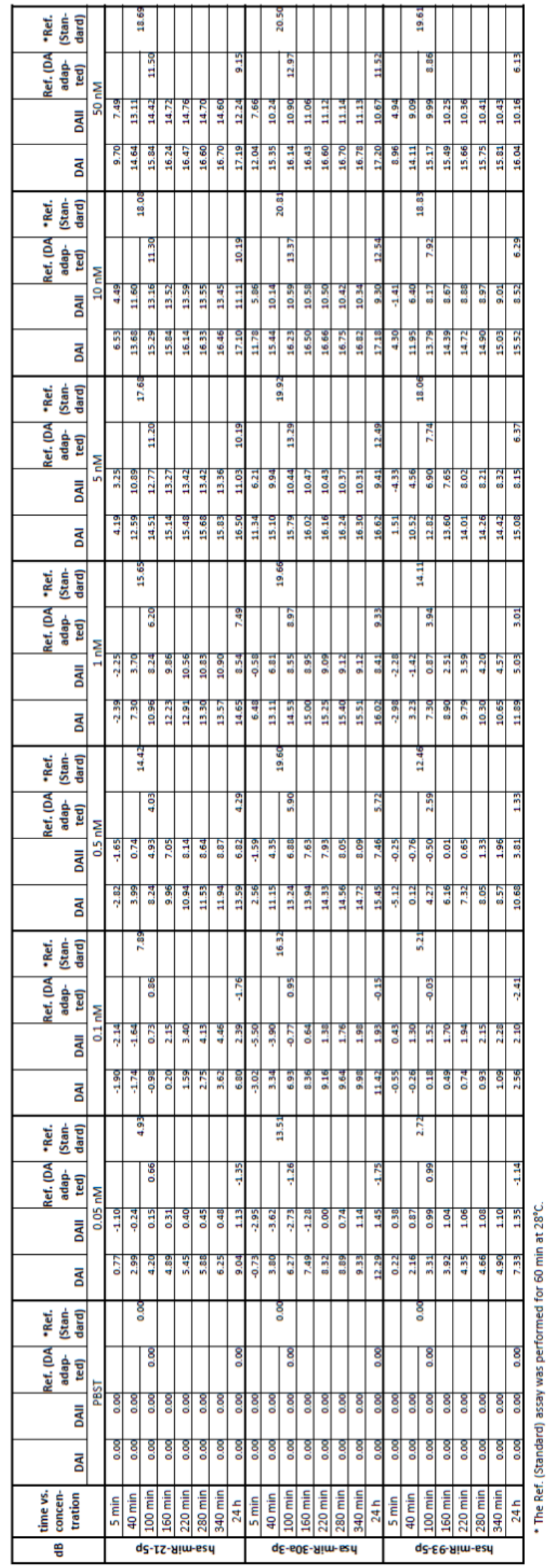




\section{Reference assay}

\section{Standard}

The miRNA hybridization and antibody binding was performed in a $200 \mu \mathrm{L}$ reaction vessel. In this assay, the capture probe-loaded microbeads (3-plex; approx. 1,000 beads per population; see above) were added together with the respective miRNAs (serial dilutions as mentioned above), the anti-DNA:RNA hybrid antibody and the secondary antibody in the reaction vessel. The standard volume of the whole reaction mix was $50 \mu \mathrm{L}$. After $1 \mathrm{~h}$ incubation at $28^{\circ} \mathrm{C}$ with vigorous shaking $(1,200 \mathrm{rpm})$, the microbeads were washed three times with PBS-T, transferred to a 96-well plate, and the fluorescence signals were analyzed with VideoScan (4 images per well).

\section{Adapted to the diffusion assay}

Since the volume and concentration ratios between the standard assay and the microchamber diffusion assay differ, the standard assay was adapted. Here, the same volume and concentration ratios were used as in the diffusion microchamber. In order to achieve a sufficient mixing of the capture probe-loaded microbeads, miRNAs and antibodies in the $200 \mu \mathrm{L}$ reaction vessel, only the total reaction volume was increased (2fold) without changing the ratios to each other. The reaction mix was incubated at $24{ }^{\circ} \mathrm{C}$ and vigorous shaking (1,200 rpm). After $100 \mathrm{~min}$ and $24 \mathrm{~h}$ incubation time, $9 \mu \mathrm{L}$ (total volume in the diffusion microchamber) were taken from the reaction mixture, washed three times with PBS-T, transferred to a 96-well plate, and the fluorescence signals were analyzed with VideoScan (4 images per well).

\section{Comparison of the microchamber diffusion assay with the reference assay}

In both diffusion approaches, the hybridization of the miRNA takes place stepwise to the capture probe loaded microbeads, which are randomly ordered on the bottom surface of the chamber. In contrast, miRNA hybridization was performed in a reaction vessel in which all molecules are exposed to the microbeads simultaneously (Fig. S6 A). 


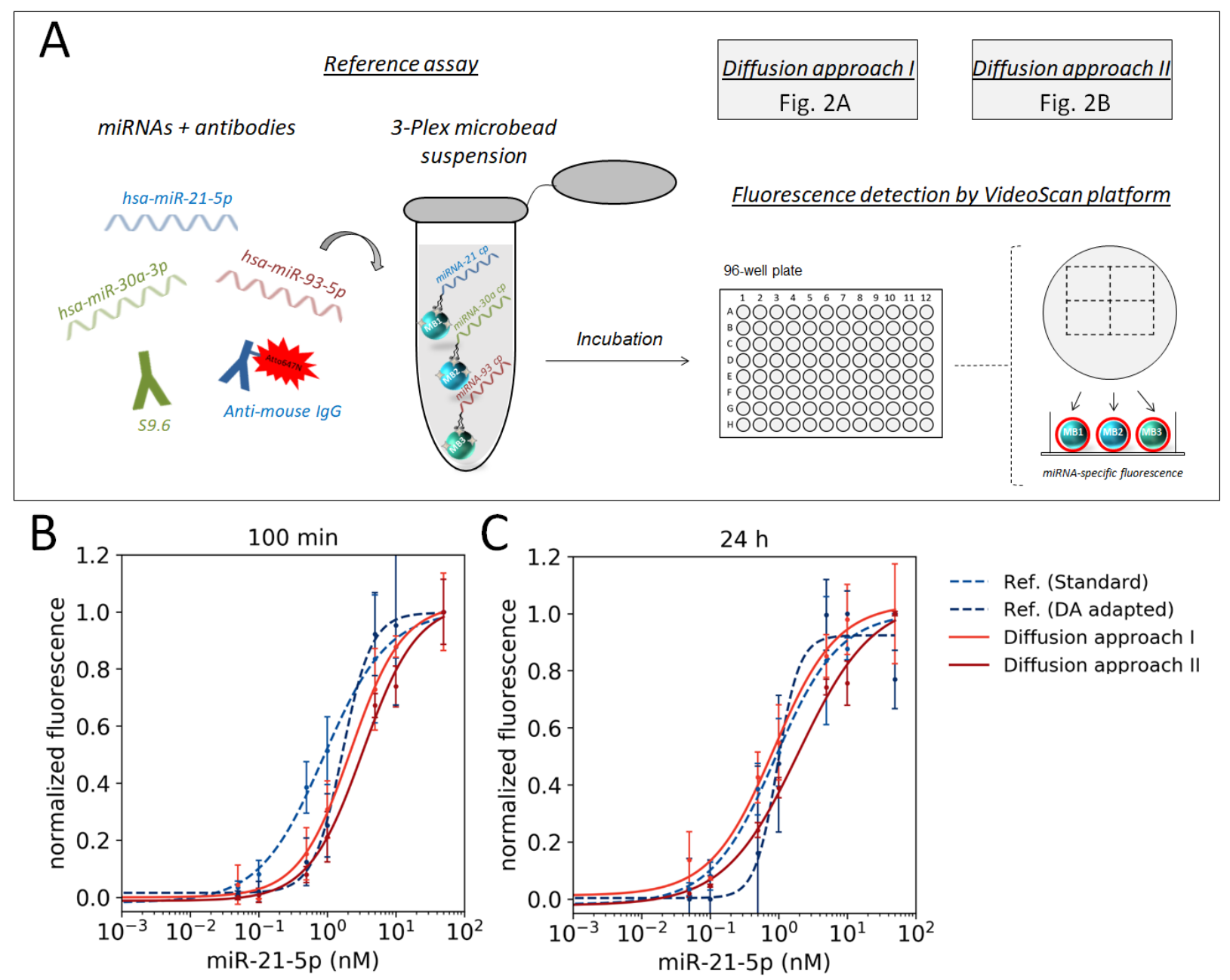

Figure S6. Comparison of the microchamber diffusion assay with the reference assay (A) Cartoon of the reference assay. The miRNA hybridization and antibody binding takes place simultaneously in one reaction vessel and the fluorescence is then measured in 96-well format by VideoScan. For the adapted reference assay, the volume and concentration ratios as well as the incubation times of the diffusion assay were used. Comparison of the concentration-response curves exemplified by miR-21-5p at timepoints (B) $100 \mathrm{~min}$ and (C) $24 \mathrm{~h}$. The standard reference assay curve is the same in panel (B) and (C). Shown are mean \pm standard deviation ( $n=3)$. Ref.(Standard) = standard reference assay. Ref.(DA adapted) $=$ reference assay adapted to the diffusion assay.

In the standard reference assay, miRNA, both antibodies and the microbeads carrying capture probes are incubated in a defined reaction volume for a defined time and then the entire reaction mixture is measured by fluorescence microscopy in 96-well format. This reference assay has also been modified by adapting it to the diffusion assay in terms of 
volume and concentration ratios, temperature and incubation times. This is referred to hereinafter as the adapted reference assay.

The comparison of the concentration-response curves is exemplified for miR-21-5p after $100 \mathrm{~min}$ in Fig. S6 B and after $24 \mathrm{~h}$ in Fig. S6 C. The curve for the standard reference assay is the same in both panels, as the incubation time is $1 \mathrm{~h}$ and the temperature $28{ }^{\circ} \mathrm{C}$. For the adapted reference assay, the diffusion assay I and II the conditions are the same. It can be seen that the $E C_{50}$ and $S D M$ values between diffusion and reference assays do not considerably differ (Table S4) and thus no substantial improvement regarding sensitivity could be achieved. The concentration-response curves of hsa-miR-30a-3p and miR-93-5p for the standard reference assay are shown in Fig. S7 and for the adapted reference assay, the diffusion assay I and II in Fig. S8. Both miRNAs show comparable results to miR-21-5p.
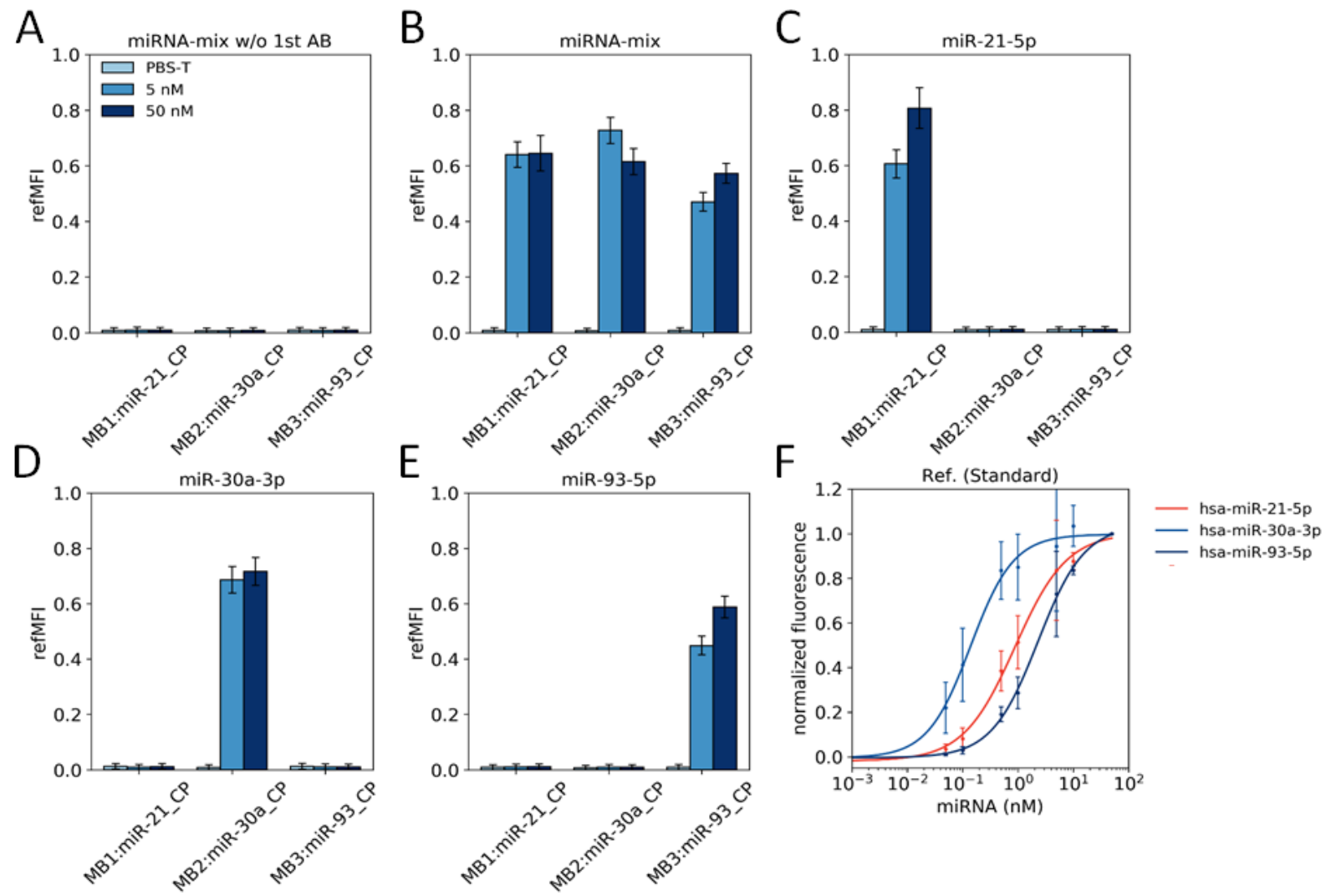

Figure S7. Bulk mixed endpoint measurement. The capture probe-loaded microbeads (3plex), miRNAs (as mixture or individually), primary and secondary antibody were mixed and incubated for $1 \mathrm{~h}$ at $28{ }^{\circ} \mathrm{C}$ with vigorous shaking. Shown are multiplex detection of (A) miRNA mixture without primary antibody, (B) miRNA mixture, (C) hsa-miR-21-5p, (D) hsa-miR-30a$3 p$, (E) hsa-miR-93-5p and $(F)$ concentration-response curves as mean \pm standard deviation of 
$n=3$ experiments. Ref. (Standard) = reference assay standard method, $M B=$ microbeads, $C P=$ capture probe.

A

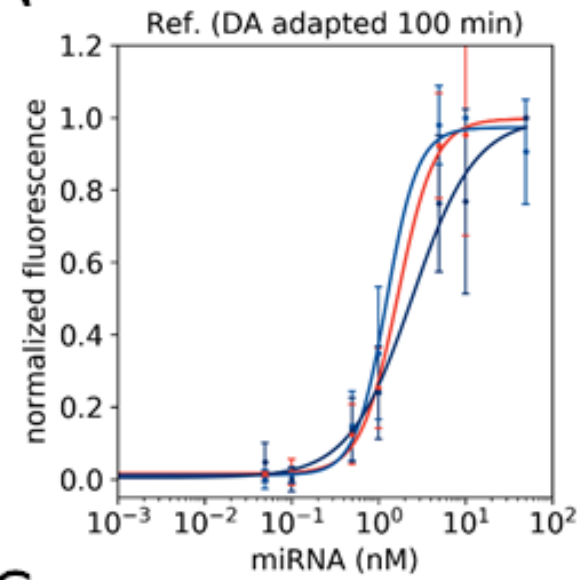

C

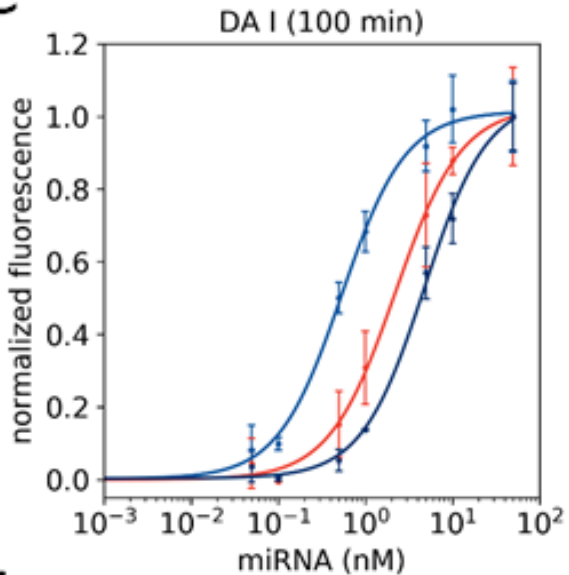

$\mathrm{E}$

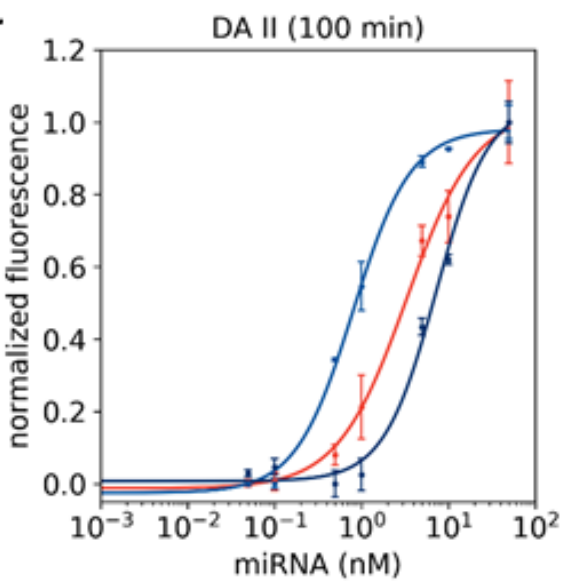

$\mathrm{B}$
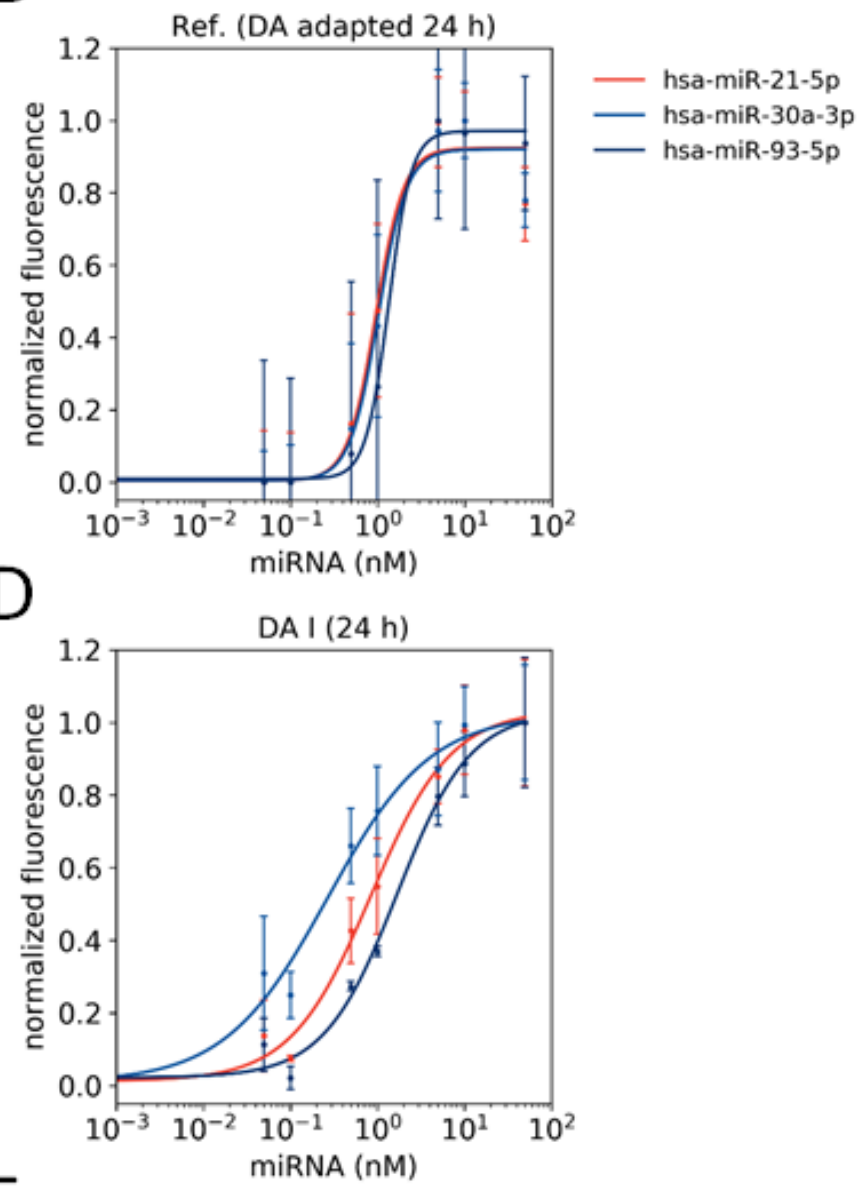

F

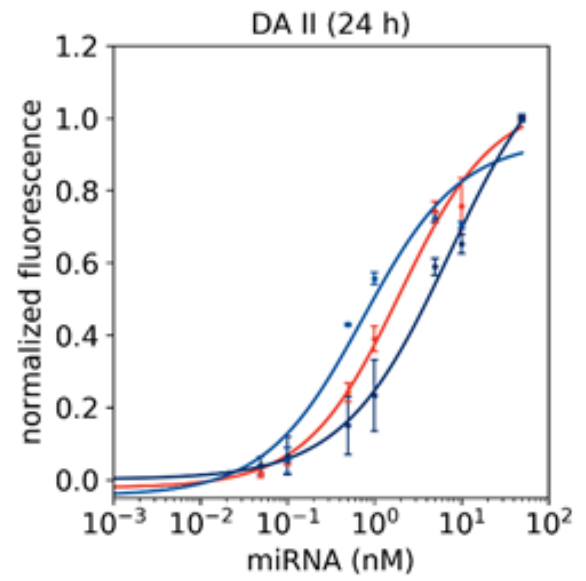

Figure S8. Comparison of the diffusion assay with the reference assay. Shown are concentration-response curves of (A) diffusion assay adapted reference assay at $100 \mathrm{~min},(B)$ diffusion assay adapted reference assay at $24 \mathrm{~h},(C)$ diffusion approach $I$ at $100 \mathrm{~min},(D)$ 
diffusion approach I at $24 \mathrm{~h}$, (E) diffusion approach II at 100 min and (F) diffusion approach II at $24 \mathrm{~h}$ as mean \pm standard deviation of $n=3$ experiments. Ref. (DA adapted $)=$ reference assay adapted to the diffusion assay, DA I/ DA II = diffusion approach I/ II

Table S4. Comparison of $E C_{50}$ and $S D M$ values of the diffusion assay and the reference assay.

\begin{tabular}{lccc}
\hline Assay & hsa-miR-21-5p & hsa-miR-30a-3p & hsa-miR-93-5p \\
\hline$E C_{50}$ (nM): & & & \\
Ref. (Standard) & $1.56 \pm 1.26$ & $0.16 \pm 0.07$ & $2.68 \pm 1.33$ \\
Ref. (DA adapted 100 min) & $1.86 \pm 0.42$ & $1.43 \pm 0.55$ & $2.69 \pm 1.09$ \\
Ref. (DA adapted 24 h) & $0.82 \pm 0.15$ & $1.04 \pm 0.35$ & $1.59 \pm 1.05$ \\
DA I (100 min) & $2.41 \pm 0.78$ & $0.54 \pm 0.05$ & $5.42 \pm 1.39$ \\
DA I (24 h) & $0.88 \pm 0.26$ & $0.26 \pm 0.02$ & $1.91 \pm 0.68$ \\
DA II (100 min) & $3.41 \pm 0.45$ & $0.81 \pm 0.10$ & $8.03 \pm 1.65$ \\
DA II (24 h) & $3.12 \pm 1.85$ & $0.81 \pm 0.16$ & $5.69 \pm 1.54$ \\
\hline SDM(nM): & & & \\
Ref. (Standard) & $0.22 \pm 0.06$ & $0.04 \pm 0.01$ & $0.67 \pm 0.22$ \\
Ref. (DA adapted 100 min) & $0.95 \pm 0.29$ & $0.76 \pm 0.27$ & $1.01 \pm 0.45$ \\
Ref. (DA adapted 24 h) & $0.57 \pm 0.29$ & $0.61 \pm 0.23$ & $0.96 \pm 0.77$ \\
DA I (100 min) & $0.77 \pm 0.39$ & $0.16 \pm 0.01$ & $1.81 \pm 0.67$ \\
DA I (24 h) & $0.21 \pm 0.12$ & $0.03 \pm 0.02$ & $0.43 \pm 0.03$ \\
DA II (100 min) & $0.91 \pm 0.19$ & $0.27 \pm 0.07$ & $2.75 \pm 0.58$ \\
DA II (24 h) & $0.33 \pm 0.13$ & $0.37 \pm 0.40$ & $1.09 \pm 0.32$ \\
\hline
\end{tabular}

